The Journal of Engineering and Exact Sciences - jCEC, Vol. 06 N. 02 (2020)

journal homepage: https://periodicos.ufv.br/ojs/jcec

doi: 10.18540/jcecvl6iss2pp0164-0178

OPEN ACCESS - ISSN: 2527-1075

\title{
CRIAÇÃO DE BANCO DE DADOS PARA SIMULAÇÃO DA PRODUÇÃO DE BIODIESEL. PARTE 1: ESTIMATIVA DE PROPRIEDADES TERMOFÍSICAS DE ÓLEOS VEGETAIS
}

\section{CREATING A DATABASE FOR SIMULATION OF BIODIESEL PRODUCTION. PART 1: ESTIMATION OF THERMOPHYSICAL PROPERTIES OF VEGETABLE OIL}

\section{G. C. R. SILVA ${ }^{1}$ e M. H. C. ANDRADE ${ }^{2}$}

${ }^{1}$ Federal University of São João del Rei, Campus Centro Oeste Dona Lindu, Divinópolis, Minas Gerais, Brazil

${ }^{2}$ Federal University of Minas Gerais, Department of Chemical Engineering, Belo Horizonte, Minas Gerais, Brazil

\begin{abstract}
"Corresponding author. Federal University of São João del Rei, Campus Centro Oeste Dona Lindu, Biochemistry, Divinopolis, Minas Gerais, Brazil, Phone: +55 37326904495

e-mail address': giselec@ufsj.edu.br(G.C.R.Silva).
\end{abstract}

\begin{tabular}{l} 
A R T I C L E I N F O \\
\hline Article history: \\
Received 2020-04-10 \\
Accepted 2020-06-17 \\
Available online 2020-06-17 \\
p a l a vr a $s-c h$ a ve \\
Óleos vegetais \\
Biodiesel \\
Propriedades termofísicas \\
Contribuição de grupos \\
Abordagem por fragmentos \\
k e y wor $d s$ \\
Vegetable oils \\
Biodiesel \\
Thermophysical properties \\
Group contribution \\
Fragment approach
\end{tabular}

\begin{abstract}
A B S T R A C T
Researches focusing on the development of biofuel production processes have increased significantly in recent years, mainly for environmental and economic issues. The identification of low-cost raw materials and the development of simulation models able to predict the conditions of production and the properties of the components may be the way to optimize this process. The knowledge of thermophysical properties of the components of the food is crucial for the results to be like the real. Thus, the objective of this study was to evaluate methods for predicting physical and thermodynamic properties in order to develop a database of the compounds involved in the biofuels production's process and to recommend appropriate methods based on accuracy and consistency of data obtained. At the end of this work, the most viable models were recommended for each property evaluated, comparing the estimated values to the experimental data available in the literature.
\end{abstract}

R E S UM O

Pesquisas com foco no desenvolvimento de processos de produção de biocombustiveis têm aumentado significativamente nos últimos anos, principalmente por questões ambientais $e$ econômicas. A busca por matérias-primas de baixo custo, bem como o desenvolvimento de modelos de simulação capazes de prever as condições da produção e as propriedades dos componentes, podem ser o caminho para aumentar o desempenho desses processos. $O$ conhecimento propriedades termofísicas dos componentes da alimentação é crucial para que os resultados sejam similares aos reais. Assim, o objetivo deste trabalho foi avaliar métodos de estimativas de propriedades termofísicas, a fim de desenvolver uma base de dados dos compostos dos óleos vegetais e recomendar os métodos adequados com base na precisão e consistência dos dados obtidos. Ao final deste trabalho recomendou-se o modelo mais viável para cada propriedade avaliada, comparando-se os valores estimados aos dados experimentais disponíveis na literatura. 


\section{INTRODUČ̃̃}

O declínio das reservas de petróleo disponíveis e regulamentações ambientais mais rigorosos têm motivado o interesse global em estudos para descoberta e aprimoramento de fontes de energia renováveis, principalmente os biocombustíveis. A composição das matérias-primas utilizadas na fabricação de bicombustível pode provocar mudanças nas suas propriedades. Isto é, o biodiesel pode apresentar um baixo desempenho em relação as propriedades de fluxo a frio pela composição química das matérias-primas (ATABANI et al., 2012), causando problemas no sistema de injeção de combustível.

A literatura revela que há um grande volume de trabalhos realizados no projeto de processos e fabricação de biocombustíveis a partir de óleos vegetais, mas os dados experimentais disponíveis para as propriedades termodinâmicas de óleos vegetais e/ou modelos de previsão são limitados (EVANGELISTA et al., 2017a; EVANGELISTA et al., 2017b; GARCIA et al., 2013; NIKITIN e POPOV, 2014; LI et al., 2016; EVANGELISTA et al., 2018)

A escassez de dados das propriedades termofísicas dos componentes do biodiesel e óleos vegetais pode estar relacionada à dificuldade em obtê-los de forma pura e na realização dos experimentos, pois essas substâncias podem se degradar em condições severas (WALLEK et al., 2013; POLING et al., 2001). Sabe-se, no entanto, que os óleos vegetais não são compostos apenas por um triacilglicerol. Óleos e gorduras podem conter dezenas de diferentes triacilgliceróis (TG), entre eles, a tripalmitina, triestearina, a trilinoleína, entre outros. Os diacilgliceróis (DG) e monoacilgliceróis (MG) também são componentes dos óleos vegetais e, possuem poucas propriedades relatadas em banco de dados, assim como os ésteres metílicos e etílicos provenientes da reação de transesterificação e/ou esterificação (WALLEK et al., 2013; CERIANI et al., 2013; NANNOOLAL et al., 2008; MOLLER et al., 2008; EVANGELISTA et al., 2018 )

Os métodos de Contribuição de Grupo (GC) têm sido utilizados nos últimos anos para estimar algumas propriedades físico-químicas de substâncias, tais como ponto de ebulição normal, propriedades críticas e fatores acêntrico (WALLEK et al., 2013; EVANGELISTA et al., 2018; GARCIA et al., 2013). O GC considera que as propriedades das moléculas dependem da natureza dos átomos e dos tipos de ligações químicas dentro das moléculas (DO CARMO et al., 2015). Além da abordagem de $\mathrm{GC}$, os métodos com princípio dos fragmentos de contribuintes (POLING et al., 2001) têm sido usados para cálculos que consideram que as propriedades das moléculas são estabelecidas a partir das contribuições de seus grupos funcionais. Essas metodologias precisam apenas de pequenas quantidades de informações sobre as substâncias para que seja obtida uma estimativa confiável. Embora exista muitos métodos na literatura, cada um deles possui premissas embutidas e limites práticos que devem ser aplicados. Portanto, a seleção dos modelos mais adequados para a previsão das propriedades termofísicas é extremamente importante para obter resultados realistas em uma simulação de processos.

\section{METODOLOGIA}

\subsection{Descrição dos modelos preditivos das propriedades termofísicas}

Para o desenvolvimento das estimativas das propriedades, foram criadas planilhas eletrônicas no MSExcel ${ }^{\circledR}$ com as sequências de equações para os cálculos dos métodos, além de serem utilizados para organizar os dados, visualizar os resultados, as tabelas e os gráficos.

Para a aplicação dos métodos de estimativa de propriedades, as moléculas dos compostos de interesse (TGs, DGs. MGs e ácido graxos-AG) foram desenhadas, os grupos funcionais identificados e quantificados.

Exemplo de cálculo: Uma molécula do triacilglicerol PLO, formado pela junção dos ácidos graxos palmítico (P), linoleico (L) e oleico (O) em uma molécula de glicerol. Sua fórmula química é representada por $\mathrm{C}_{55} \mathrm{H}_{100} \mathrm{O}_{6}$. Escolheu-se este, por ser um triacilglicerídeo misto composto com os principais ácidos graxos presentes nos óleos vegetais. A Figura 1 representa a molécula do triacilglicerol PLO.

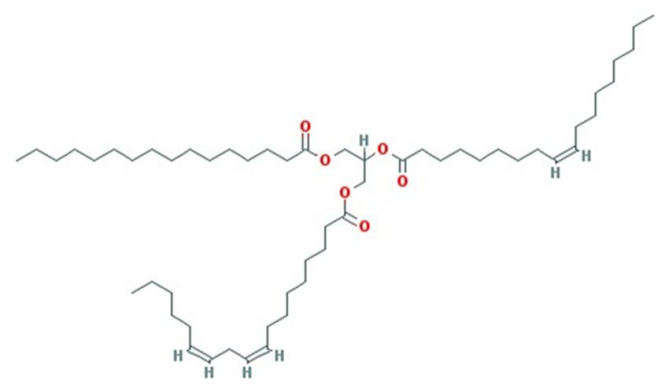

Figura 1 - Estrutura química do triacilglicerol PLO.

A partir dessa estrutura molecular foi feita a contagem dos grupos presentes na molécula (Tabela 1) para os métodos de contribuição de grupos, JOBACK e Reid (1987) e Constantinou e GANI (1994).

Tabela 1 - Ocorrência dos grupos para o método de JOBACK e GANI para o triacilglicerídeo PLO.

\begin{tabular}{cccc}
\hline Grupos & Contagem & $\begin{array}{c}\text { Grupos } \\
\text { GANI }\end{array}$ & Contagem \\
JOBACK & $\mathbf{3}$ & $\mathbf{C H}_{3}$ & 3 \\
$\mathbf{C H}_{3}$ & 42 & $>\mathbf{C H}_{2}$ & 40 \\
$>\mathbf{C H}_{2}$ & 4 & $>\mathbf{C H}-$ & 1 \\
$>\mathbf{C H}-$ & 3 & $-\mathbf{C H}_{2}$-COO-(C) & 2 \\
$-\mathbf{C O O}-$ & $-\mathbf{C O O}-$ & 1 \\
$=\mathbf{C H}-$ & 3 & $-\mathbf{C H}=\mathbf{C H}-$ & 3 \\
\hline
\end{tabular}

Cada um destes grupos, dependendo da propriedade a ser calculada e método adotado, tem um valor de contribuição. Assim, com a contagem feita, é possível utilizar as equações dos métodos com segurança para predizer a propriedade de interesse. Esse procedimento de cálculo foi feito para todas as moléculas necessárias para a simulação. 
A descrição dos métodos de contribuição de grupos e métodos baseados em fragmentos analisados neste trabalho é apresentada a seguir, assim como as equações necessárias aos cálculos.

Temperatura normal de ebulição $\left(\mathrm{T}_{\mathrm{b}}\right)$ : é a temperatura na qual a pressão de vapor se iguala à pressão externa ou atmosférica. Neste trabalho, foram analisados os métodos de contribuição de grupo de Joback e Reid (1987) e Constantinou e Gani (1994).

Constantinou e Gani (1994) desenvolveram um método avançado de contribuição de grupos baseado nos grupos do modelo termodinâmico Universal Functional Activity Coefficient (UNIFAC) (FREDENSLUND et al., 1975). Além disso, os autores adicionaram as contribuições ditas de "segunda ordem" que permitem diferenciar moléculas de isômeros, moléculas que têm os mesmos grupos, localizados em conjunto ou não, estruturas de ressonância, entre outros (POLING et al., 2001).

As equações dos modelos JOBACK e GANI para estimativa de $T_{b}$ são representadas pelas equações 1 e 2 .

\section{JOBACK:}

$T_{b}=198+\sum_{k} N_{k}\left(T_{b k}\right)$

\section{GANI:}

$$
T_{b}=204,359 \times \ln \left(\sum_{k} N_{k} T_{b k}\right)
$$

Em que $\mathrm{N}_{\mathrm{k}}$ representa o número de grupos do tipo $\mathrm{k}$ e $\mathrm{T}_{\mathrm{bk}}$ a contribuição para a temperatura normal de ebulição dos grupos $\mathrm{k}$.

Além dos métodos descritos acima, este trabalho empregou a abordagem semi-empírica proposta por Zong et al. (2010a e 2010b), em que o cálculo da temperatura de ebulição dos TGs, DGs e MGs é realizado com base em fragmentos dos constituintes. Para este propósito, uma relação entre os valores de temperatura de ebulição de cada um dos fragmentos dos TGs (ácidos graxos e glicerol) e da pressão foi feita. Portanto, os dados experimentais (FALLEIRO et al., 2012 e DDB, 2019) sobre a temperatura de cada um dos fragmentos foram ajustados à Equação 3.

$$
\begin{aligned}
& \text { ZONG } \\
& T_{b}^{a}=a \times \ln (P)+b
\end{aligned}
$$

Onde a e b são os parâmetros de ajuste de cada fragmento, $\mathrm{T}_{\mathrm{b}}{ }^{\mathrm{a}}$ é a temperatura de ebulição do fragmento a $(\mathrm{K})$ e P é a pressão de vapor $(\mathrm{mmHg})$. Sabendo o valor de $\mathrm{T}_{\mathrm{b}}{ }^{\mathrm{a}}$ para cada um dos fragmentos, foi possível calcular o valor da $\mathrm{T}_{\mathrm{b}}{ }^{\mathrm{TG}}$ usando a Equação 4:

$$
T_{b}^{T G}=\frac{\sum N_{F r a g, a} \times T_{b}^{a}}{3}
$$

Sendo, $\mathrm{T}_{\mathrm{b}}{ }^{\mathrm{TG}}$ temperatura normal de ebulição do triacilglicerídeo $(\mathrm{K})$ e $\mathrm{N}_{\text {Frag }}$ o número de fragmentos de a.

Propriedades críticas $\left(\mathrm{P}_{\mathrm{c}}, \mathrm{V}_{\mathrm{c}}, \mathrm{T}_{\mathrm{c}}\right.$ e $\left.\mathrm{Z}_{\mathrm{c}}\right)$ : As propriedades críticas, isto é, pressão $\left(\mathrm{P}_{\mathrm{c}}\right)$, volume $\left(\mathrm{V}_{\mathrm{c}}\right)$, temperatura $\left(\mathrm{T}_{\mathrm{c}}\right) \mathrm{e}$ fator de compressibilidade $\left(\mathrm{Z}_{\mathrm{c}}\right)$, são parâmetros utilizados em muitas correlações termodinâmicas, volumétricas e de transporte baseadas na teoria dos estados correspondentes para estimar propriedades de gases e líquidos. Portanto, são dados importantes nos estudos de otimização de processos.
Segundo Poling et al. (2001), a determinação experimental dessas propriedades é difícil, pois pode ocorrer degradação química em altas temperaturas, especialmente para componentes de cadeias longas.

Nikitin et al. (1993) definiram o ponto crítico do glicerol através de uma metodologia não convencional chamada "pulseheating". Esta é única medida experimental disponível na literatura. As propriedades críticas obtidas foram $T_{c}=850 \mathrm{~K}$ e $\mathrm{P}_{\mathrm{c}}=75$ bar. Como o glicerol inicia sua degradação térmica a 510 K (DIPPR, 2019), os parâmetros críticos desta molécula podem ser incluídos em cálculos preditivos

Os métodos de estimativa avaliados neste trabalho foram: Constantinou e Gani (1994), Ambrose (1980), Joback e Reid (1987) e Lydersen (1955). O primeiro método necessita apenas da informação da estrutura do composto, enquanto os demais, além de serem baseados na estrutura molecular do composto, necessitam dos valores da massa molar e temperatura de ebulição. Os métodos são representados pela Equação 5 a Equação 16.

\section{JOBACK}

$$
\begin{aligned}
& T_{c}=T_{b}\left[0,584+0,965 \sum_{k} N_{k}\left(T_{c k}\right)-\right. \\
& \left.\left(\sum_{k} N_{k}\left(T_{c k}\right)\right)^{2}\right]^{-1} \\
& P_{c}=\left[0,113+0,0032 N_{a t}-\sum_{k} N_{k}\left(P_{c k}\right)\right]^{-2} \\
& V_{c}=17,5+\sum_{k} N_{k}\left(V_{c k}\right)
\end{aligned}
$$

\section{GANI}

$$
\begin{aligned}
& T_{c}=181,28 \times \ln \left(\sum_{k} N_{k} T_{c k}\right) \\
& P_{c}=\left[\sum_{k} N_{k} P_{c k}+0,10022\right]^{-2}+1,3705 \\
& V_{c}=\left(\sum_{k} N_{k}\left(V_{c k}\right)-0,00435\right) \times 1000,0
\end{aligned}
$$

AMBROSE:

$$
\begin{aligned}
& T_{c}=T_{b}\left[1+\left(1,242+\sum_{k} N_{k}\left(T_{c k}\right)\right)^{-1}\right] \\
& P_{c}=M_{M}\left[0,339+\sum_{k} N_{k}\left(T_{c k}\right)\right]^{-2} \\
& V_{c}=40+\sum_{k} N_{k}\left(V_{c k}\right)
\end{aligned}
$$

\section{LYDERSEN:}

$$
\begin{aligned}
T_{c} & =\frac{T_{b}}{0,567+\sum N_{k}\left(T_{c k}\right)-\left(\sum N_{k}\left(T_{c k}\right)\right)^{2}} \\
P_{c} & =\frac{M_{M}}{\left(0,34+\sum N_{k}\left(P_{c k}\right)\right)^{2}} \\
V_{c} & =40+\sum N_{k}\left(V_{c k}\right)
\end{aligned}
$$

nas quais $T_{c k}, P_{c k}$ e $V_{c k}$, são as contribuições de cada grupo funcional e que variam numericamente dependendo do método de contribuição de grupos. $\mathrm{N}_{\mathrm{at}}$ é o número de átomos na molécula, na equação de pressão crítica para o método de JOBACK. $\mathrm{M}_{\mathrm{M}}$ a massa molar, $\mathrm{N}_{\mathrm{k}}$ é o número de vezes que o grupo " $k$ " aparece na molécula. $\mathrm{T}_{\mathrm{c}}$ é dado em Kelvin, $\mathrm{P}_{\mathrm{c}} \mathrm{em}$ bar

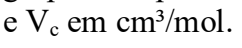

Fator acêntrico $(\omega)$ : $\mathrm{O}$ fator acêntrico foi introduzido por Kenneth Sunborn Pitzer em 1955. É um número conceitual, em 
que é empregado nas equações de estado cúbicas. Está relacionado com a esfericidade do campo de força da molécula (POLING et al., 2001). Portanto, está ligado também à polaridade da molécula, quanto mais elevado o valor maior a polaridade. Originalmente foi definido pela Equação 17:

$$
\omega=-\log \left[\lim _{\left(\frac{T}{T_{c}}\right)=0,7}\left(\frac{P_{v p}}{P_{c}}\right)\right]-1,0
$$

Sendo $\mathrm{P}_{\mathrm{vp}}$ a pressão de vapor, $\mathrm{T}_{\mathrm{c}}$ a temperatura crítica $\mathrm{e}$ $\mathrm{P}_{\mathrm{c}}$ a pressão crítica.

A definição particular da equação (Equação 17) surgiu porque os gases monoatômicos ( $\mathrm{Ar}, \mathrm{Kr}, \mathrm{Xe}$ ) tem $\omega \sim 0$, com exceção dos gases nobres (He, $\mathrm{Ne}$ ) e alguns outros (por exemplo, $\mathrm{Rn}$ ), todas as outras espécies têm valores positivos até 1,5. Para obter valores de $\omega$, pela definição, é preciso conhecer as constantes $T_{c}, P_{c}$ e a propriedade $P_{v p}$ na temperatura reduzida, $\mathrm{T} / \mathrm{T}_{\mathrm{c}}=0,7$.

Dois métodos foram selecionados neste trabalho para avaliação, a regra de Pitzer (PITZER) e Kesler- Lee (LEE).

Pitzer (1995) apresenta a Equação 18 para cálculo do fator acêntrico:

$$
Z_{c}=0,291-0,080 \omega
$$

Segundo Poling et al. (2001), a Equação 18 pode ser usada para predizer o fator acêntrico de substâncias associativas e substâncias fortemente polares. No entanto, os autores destacam que se os parâmetros críticos não foram adequadamente estimados para a substância em questão não há garantia da precisão da propriedade desejada.

Um conjunto de equações foi desenvolvido por Kesler e Lee (1976) para avaliar propriedades de compostos químicos como a massa molecular, temperatura normal de ebulição, pressão crítica, temperatura crítica e fator acêntrico. Para este último, as seguintes correlações foram desenvolvidas (Equação 19 e Equação 20). Tais modelos dependem da temperatura de ebulição reduzida $\left(\mathrm{T}_{\mathrm{br}}=\mathrm{T}_{\mathrm{b}} / \mathrm{T}_{\mathrm{c}}\right)$ e do fator de caracterização de Watson $\left(\mathrm{K}_{\mathrm{W}}\right)$.

$$
\begin{gathered}
\text { Para } \mathrm{T}_{\mathrm{br}}>0,8: \\
\omega=-7,904+0,1352 \mathrm{~K}_{\mathrm{w}}-0,007465 \mathrm{~K}_{\mathrm{w}}^{2}+ \\
8,359 \mathrm{~T}_{\mathrm{br}}+\frac{\left(1,408-0,01063 \mathrm{~K}_{\mathrm{w}}\right)}{\mathrm{T}_{\mathrm{br}}} \\
\text { Para } \mathrm{T}_{\mathrm{br}}<0,8: \\
\omega=\frac{-\ln P_{c}-5,92714+\frac{6,09648}{T_{b r}}+1,28862 \ln T_{b r}-0,169347 T_{b r}^{6}}{15,2518-\frac{15,687}{T_{b r}}-13,4721 \ln T_{b r}+0,43577 T_{b r}^{6}}
\end{gathered}
$$

O fator de Watson pode ser definido pela Equação 21:

$$
K_{w}=\frac{M T_{b}^{1 / 3}}{d_{15^{\circ} \mathrm{C}}}
$$

Onde $\mathrm{MT}_{\mathrm{b}}$ é a média da temperatura normal de ebulição dada em ${ }^{\circ} \mathrm{R}$ e d é a densidade relativa medida a $15^{\circ} \mathrm{C}$.

Entalpia e energia de Gibbs de formação de gás no estado padrão: A determinação da entalpia padrão de formação $\Delta \mathrm{H}_{\mathrm{f}}^{0}$ é importante por fornecer a entalpia padrão de reação para a formação de um mol de uma substância a partir dos elementos químicos que a constituem, em seus estados de referência.
Portanto, como neste trabalho deseja-se criar um banco de dados para ser usado em simulação e otimização de processos de produção de biocombustíveis, o conhecimento dessas propriedades deve ser considerado.

Similarmente à entalpia padrão de formação, define-se a energia de Gibbs padrão de formação, $G_{f}^{0}$, como sendo a energia de Gibbs padrão de reação para a formação de um mol de uma substância a partir dos elementos químicos que a constituem em seus estados de referência. Calcula-se a energia de Gibbs de reação a partir das energias de Gibbs padrão de formação das espécies envolvidas. Segundo Atkins (2012), as energias de Gibbs podem ser obtidas a partir de dados calorimétricos (entalpia e entropia, a partir de capacidades caloríficas), constantes de equilíbrio em reações químicas, medidas eletroquímicas e por dados de espectroscopia, quando na fase gasosa.

Raramente valores para o cálculo da energia de Gibbs da reação são encontrados. Métodos de contribuições de grupos como os de Joback e Reid (1987), Benson et al. (1969), Constantinou e Gani (1994) e outros, têm sido de empregados para a predição de estimativas confiáveis de valores de entalpias de formação, entropias absolutas e energias de Gibbs.

Escolhendo os grupos atômicos e moleculares de primeira e segunda de ordem de um composto, Constantinou e Gani (1994) obtiveram contribuições do grupo para $\mathrm{G}_{\mathrm{f}}(298,15$ $\mathrm{K})$ e $\mathrm{H}_{\mathrm{f}}(298,15 \mathrm{~K}$ ) através das equações (Equação 22 e Equação 23).

\section{GANI}

$$
\begin{aligned}
& H_{f}^{0}=10,835+\sum_{k} N_{k}\left(H_{f k}\right) \\
& G_{f}^{0}=-14,828+\sum_{k} N_{k}\left(G_{f k}\right)
\end{aligned}
$$

Em que $\mathrm{N}_{\mathrm{k}}$ representa o número de grupos e $\mathrm{H}_{\mathrm{fk}}$ e $\mathrm{G}_{\mathrm{fk}} \mathrm{a}$ contribuição do grupo para entalpia e energia de Gibbs, respectivamente.

O método de Benson et al. (1969) pode ser utilizado também para a predição de entalpias de formação e entropias absolutas de compostos químicos em fase gasosa no estado padrão. Apenas átomos com valência maior que um são determinados por este método. Os grupos são ligados a átomos chave com nomenclatura específica (Equação 24, Equação 25 e Equação 26). Por exemplo, o grupo C-(C)(H $)_{3}$ é uma referência ao átomo de carbono (átomo chave) ligado a outro carbono e três hidrogênios.

$$
\begin{aligned}
\text { BENSON } & \\
\Delta H_{f}^{0}(298,15 K) & =\sum_{k} N_{k}\left(\Delta H_{f k}^{0}\right) \\
\Delta G_{f}^{0}(298,15 K) & =\Delta H_{f}^{0}(298,15 K)-298,15 \Delta S_{f}^{0} \\
\Delta S_{f}^{0}(298,15 K) & =\sum_{k} N_{k}\left(\Delta S_{f k}^{0}\right)-R \ln \sigma+\ln \eta
\end{aligned}
$$

Onde $\sigma$ é a simetria da molécula e $\eta$ o número de isômeros. $\mathrm{R}$ é a constante universal dos gases.

O método de Joback e Reid (1987) é uma extensão do método de Lydersen (1955). É um método que não utiliza contribuições para interações entre os grupos e utiliza contribuições aditivas. Segundo os autores, não é um método de 
alta precisão, mas funciona bem para muitas espécies químicas. Dentre essas propriedades, as de interesse para este trabalho são as estimativas de energia de Gibbs de formação padrão e entalpia de formação padrão. A Equação 27 e Equação 28 são utilizadas por esse método para estimativa destas duas propriedades.

$$
\begin{aligned}
& \text { JOBACK } \\
& \begin{array}{l}
H_{f}^{0}=68,29+\sum_{k} N_{k}\left(H_{f k}\right) \\
G_{f}^{0}=53,88+\sum_{k} N_{k}\left(G_{f k}\right)
\end{array}
\end{aligned}
$$

Em que $\mathrm{N}_{\mathrm{k}}$ representa o número de grupos, $\mathrm{H}_{\mathrm{fk}}$ contribuição para a entalpia de formação e $\mathrm{G}_{\mathrm{fk}}$ contribuição para a energia de Gibbs de formação dos grupos k.

Ressalta-se a similaridade da Equação 22 e Equação 27, para o cálculo da entalpia de formação nos métodos de GANI e JOBACK, respectivamente. A principal diferença nessas equações encontra-se na identificação do grupo k. Sabe-se que o método de GANI considera interações de segunda ordem, consequentemente, os grupos identificados por este método pode ser diferente do obtido por JOBACK. De forma semelhante, acontece na Equação 23 e Equação 28 para o cálculo da energia de Gibbs.

Pressão de vapor $\left(\mathrm{p}_{\mathrm{vap}}\right)$ : Zong et al. (2010a) aplicaram o método baseado em fragmento e a equação de ClausiusClapeyron para estimar pressões de vapor dos TGs, através da Equação 29. Devido à falta de dados experimentais para pressões de vapor de TGs insaturados, a abordagem à base de fragmentos assume que as cadeias de ácidos graxos saturados e insaturados possuem pressões de vapor idênticas, quando se tem o mesmo número de átomos de carbono.

$$
\begin{aligned}
& \text { ZONG } \\
& \log P(T)=\frac{-\Delta G_{\theta}^{\text {vap }}}{R \theta \ln 10}+\frac{\Delta H_{\theta}^{\text {vap }}}{R \theta \ln 10}\left(\frac{1}{\theta}-\frac{1}{T}\right)
\end{aligned}
$$

onde $\mathrm{P}$ é a pressão de vapor $(\mathrm{Pa}), \mathrm{T}$ é a temperatura absoluta $(\mathrm{K}), \mathrm{R}$ é a constante dos gases, $\theta$ é a temperatura de referência $(\theta=298,15 \mathrm{~K}), \Delta \mathrm{H}_{\theta}{ }^{\mathrm{vap}}$ é a entalpia de vaporização na temperatura de referência $\theta$, e $\Delta \mathrm{G}_{\theta}$ vap é a energia de Gibbs de vaporização na temperatura de referência $\theta$.

Ceriani et al. (2013) propuseram um método de contribuição de grupo para estimar as pressões de vapor e os calores de vaporização em função da temperatura de líquidos orgânicos encontrados nas indústrias de óleos e biocombustíveis. A regressão dos parâmetros foi baseada em um extenso banco de dados, composto por ácidos graxos, ésteres de metila, etila, propila e butila, tri-, di- e monoacilgliceróis e hidrocarbonetos. Esta metodologia melhorou as previsões obtidas pelo método de Ceriani e Meirelles (2004), devido à inclusão de novos dados experimentais de ésteres e acilgliceróis (além de hidrocarbonetos) e pontos críticos. A Equação 30 mostra o cálculo da pressão de vapor $\left(\mathrm{P}_{\mathrm{vap}}\right)$ em $\mathrm{Pa}$ e temperatura em K.

$$
\begin{aligned}
& \text { CERIANI } \\
& \ln \left(P^{\text {vap }}\right)=A+\frac{B}{T}+C \cdot \ln (T) \\
& A=\sum_{k} N_{k}\left(A_{1 k}+M_{M} \cdot A_{2 k}\right)+\left(s_{0}+\right. \\
& \left.N_{C S} \cdot s_{1}\right)+\alpha\left(f_{o}+N_{C} \cdot f_{1}\right)
\end{aligned}
$$

$$
\begin{aligned}
& B=\sum_{k} N_{k}\left(B_{1 k}+M_{M} \cdot B_{2 k}\right)+\beta\left(f_{o}+\right. \\
& \left.N_{C} \cdot f_{1}\right) \\
& C=\sum_{k} N_{k}\left(C_{1 k}+M_{M} \cdot C_{2 k}\right)
\end{aligned}
$$

onde $\mathrm{N}_{\mathrm{k}}$ é o número de grupos $\mathrm{k}$ na molécula, $\mathrm{MM}$ é a massa molecular do componente, $\mathrm{NC}_{\mathrm{s}}$ é o número de carbonos da parte alcoólica dos ésteres metílicos e etílicos $\left(\mathrm{NC}_{\mathrm{s}}\right.$ é igual a 1 e 2, respectivamente), $\mathrm{NC}$ é o número total de átomos de carbono na molécula. $\mathrm{A}_{1 \mathrm{k}}, \mathrm{B}_{1 \mathrm{k}}, \mathrm{C}_{1 \mathrm{k}}, \mathrm{A}_{2 \mathrm{k}}, \mathrm{B}_{2 \mathrm{k}}, \mathrm{C}_{2 \mathrm{k}}, \alpha, \beta, \mathrm{s}_{0}, \mathrm{~s}_{1}, \mathrm{f}_{0}$ e $f_{1}$ são os parâmetros obtidos pela regressão.

Ceriani et al. (2013) melhoraram o modelo de previsão da pressão de vapor e calor de vaporização de compostos orgânicos até o ponto crítico utilizando os mesmos conjuntos de grupos funcionais como proposto em Ceriani e Meirelles (2004), mas com uma outra dependência da temperatura. A proposta dos autores foi consequência dos trabalhos de Ceriani et al. (2009) e Su et al. (2011), os quais indicaram a limitação da equação de determinação das entalpias de vaporização a um intervalo de temperatura.

Entalpia de vaporização $\left(\mathrm{H}_{\text {vap }}\right)$ : Ceriani et al. (2009) desenvolveram um modelo para prever o calor de vaporização a partir da equação Clausius-Clapeyron (Equação 31) e o método de contribuição de grupo de Ceriani e Meirelles (2004) (Equação 32). Ao substituir a expressão da pressão de vapor na equação Clausius-Clapeyron e algumas manipulações, obtevese uma equação para $\Delta \mathrm{H}_{\text {vap }}$ em função da temperatura (Equação 33).

$$
\begin{aligned}
& \frac{d P_{i}^{\text {vap }}}{d T}=\frac{P_{i}^{\text {vap }} \Delta H_{i}^{\text {vap }}}{R T^{2}} \\
& P_{i}^{\text {vap }}=\exp \left(A_{i}+\frac{B_{i}}{T^{1,5}}-C_{i} \ln T-D_{i} T\right) \\
& \Delta H_{i}^{\text {vap }}=-R\left(\frac{1,5 B_{i}}{\sqrt{T}}+C_{i} T+D_{i} T^{2}\right)
\end{aligned}
$$

onde $\mathrm{R}$ é a constante universal dos gases e $\mathrm{B}_{\mathrm{i}}, \mathrm{C}_{\mathrm{i}}$ e $\mathrm{D}_{\mathrm{i}}$ são os parâmetros do mesmo grupo de contribuição usados para estimar a pressão de vapor no método de Ceriani e Meirelles (2004). Em condições de alta temperatura e alta pressão de vapor, a condição de gás ideal feita na equação (Equação 33) não é válida. Portanto, Ceriani et al. (2009) incluíram um termo de correção, que originou a Equação 34.

$$
\begin{aligned}
\Delta H_{i}^{\text {vap }}=-R\left(\frac{1,5 B_{i}}{\sqrt{T}}+C_{i} T\right. & \\
& \left.+D_{i} T^{2}\right)\left(1-\frac{T_{c}^{3} P_{i}^{\text {vap }}}{T^{3} P_{c}}\right)^{0,5}
\end{aligned}
$$

onde $\mathrm{P}_{\mathrm{i}}^{\text {vap }}$ é a pressão de vapor do componente $\mathrm{i}$ e Tc e Pc são a temperatura crítica e pressão crítica, respectivamente.

Ceriani et al. (2013) fizeram alterações do modelo anterior de predição do calor de vaporização para que atingisse uma maior gama de compostos. Assim, os autores estudaram diversos compostos disponíveis nos bancos de dados para que os parâmetros da equação para o cálculo do calor de vaporização fossem atualizados.

Pitzer (1955) utilizaram uma equação linear para estimar o calor de vaporização, $\Delta \mathrm{H}_{\text {vap, }}$ como função da temperatura reduzida (Tr) e fator acêntrico $\omega$. Su et al. (2011) derivaram essa expressão em uma equação analítica, através de uma aproximação da correlação de $0,6<\operatorname{Tr}<1,0$ (Equação 35). 


$$
\begin{aligned}
\frac{\Delta H_{v a p}}{R T_{c}}=7,08(1 & \left.-T_{r}\right)^{0,354} \\
& +10,95 \omega\left(1-T_{r}\right)^{0,456}
\end{aligned}
$$

Basarova e Svoboda (1995) aplicaram outro método de contribuição de grupo para estimar o calor de vaporização para diversas substâncias em função da temperatura reduzida (Equação 36).

$$
\Delta H_{\text {vap }}=A\left(1-T_{r}\right)^{\alpha} \exp \left(-\alpha T_{r}\right)
$$

onde $\Delta$ Hvap representa o calor de vaporização, $\operatorname{Tr}$ a temperatura reduzida, e A e $\alpha$ são parâmetros de contribuição do grupo.

Para prever a entalpia de vaporização, Zong et al. (2010a) utilizaram a Equação 29, em que o calor de vaporização aparece da correlação de pressão de vapor pela equação de Antoine. Não foi avaliada a dependência da temperatura com o calor de vaporização.

Densidade ( $\rho$ ): Halvorsen et al. (1993) utilizaram a equação modificada de Rackett para estimar a densidade de óleos vegetais líquidos. Primeiramente foi estimada a densidade da mistura líquida de ácidos graxos livres e, em seguida, um fator de correção foi adicionado para descrever a forma dos TGs (Equação 37). Não foram apresentados fatores de correção para os DGs e MGs.

$$
\rho=\frac{\sum x_{i} M M_{i}}{R\left(\frac{\sum x_{i} T_{c i}}{P_{c i}}\right)\left(\sum x_{i} Z_{R A i}\left[1+(1-T r)^{\frac{2}{7}}\right]\right.}+F_{c}
$$

onde $\mathrm{x}_{\mathrm{i}}, \mathrm{MM}_{\mathrm{i}}, \mathrm{T}_{\mathrm{ci}}, \mathrm{P}_{\mathrm{c}}, \mathrm{e} \mathrm{Z}_{\mathrm{RAi}}$ são frações molares, massa molecular, temperatura crítica, pressão crítica e o parâmetro de Rackett, respectivamente, de cada ácido graxo i. $T_{\mathrm{r}}$ é a temperatura reduzida. A densidade é dada $\mathrm{em} \mathrm{kg} / \mathrm{m}^{3}$.

Zong et al. (2010a) propuseram a abordagem baseada em fragmentos para estimar o volume molar de cada fragmento com dependência com a temperatura e, em seguida, estimaram o volume molar do líquido com base na composição e na contribuição de cada fragmento (Equação 38).

$$
v^{L}=\sum_{A} N_{\text {frag, } A} v_{A}^{L}(T)
$$

onde $v_{\mathrm{A}}{ }^{\mathrm{L}}$ é o volume molar do líquido de cada fragmento e $\mathrm{N}_{\text {frag,A }}$ é o número de fragmentos $\mathrm{A}$ em um componente.

Ihmels e Gmehling (2003) estenderam o método de contribuição de grupos desenvolvido por Elbro et al. (1991) para prever as densidades de compostos líquidos puros (Equação 39).

$$
\rho=\frac{M M}{v}=\frac{M M}{\sum n_{i} \Delta v_{i}}
$$

sendo MM a massa molecular e $v$ o volume molar.

Capacidade calorífica ( $\left.\mathrm{c}_{\mathrm{P}}\right)$ : Zong et al. (2010a) também aplicaram seu método baseado em fragmentos para estimar a capacidade calorífica dos TGs, DGs e MGs. Os fragmentos de TGs, DGs e MGs foram expressos como equações lineares dependentes da temperatura (Equação 40). Os fragmentos de ácido graxos insaturados foram assumidos idênticos.

$$
C_{p}^{L}=\sum_{A} N_{\text {frag }, A} C_{p, A}^{L}(T)
$$

Onde $\mathrm{N}_{\text {frag,A }}$ é o número de fragmentos A no componente e $\mathrm{C}_{\mathrm{p}, \mathrm{A}} \mathrm{L}^{\mathrm{L}}$ a contribuição da capacidade calorífica do fragmento no componente $\mathrm{A}(\mathrm{J} / \mathrm{kmol} . \mathrm{K})$.

Ceriani et al. (2009) estenderam seu método de contribuição de grupos anteriormente utilizado para a previsão de pressão de vapor de líquidos orgânicos para desenvolver um modelo de capacidade de calor com o mesmo conjunto de grupos funcionais além de uma nova relação linear em função da contribuição do grupo (Equação 41).

$$
C_{p i}^{L}=\sum_{k} N_{k}\left(A_{k}+B_{k} T\right)
$$

onde $\mathrm{N}_{\mathrm{k}}$ é o número de grupos $\mathrm{K}$ na molécula, $\mathrm{A}_{\mathrm{k}}$ e $\mathrm{B}_{\mathrm{k}}$ são obtidos a partir dos parâmetros de regressão.

Morad et al. (2000) estimaram as capacidades caloríficas para TGs e óleos vegetais, primeiramente pela aplicação da equação Rowlinson-Bondi (REID et al., 1987). Pela Equação 42 estimaram as capacidades de calor dos ácidos graxos puros. Em seguida, adicionaram um fator de correção, com base no trabalho de Halvorsen et al (1993), para predizer a densidade dos triacilgliceróis. Resultando em uma expressão com dependência da massa molecular do óleo (Equação 43).

$$
\begin{aligned}
\frac{C_{p(A G)}-C_{p(A G)}{ }^{0}}{R} & =1,45+0,45\left(1-T_{r}\right)^{-1} \\
& +0,25 \omega[17,11 \\
& +25,2\left(1-T_{r}\right)^{1 / 3} T_{r}^{-1} \\
& \left.\left.+1,742\left(1-T_{r}\right)^{-1}\right]\right]
\end{aligned}
$$

onde $\mathrm{c}_{\mathrm{p}(\mathrm{AG})}$ é o calor específico líquido dos ácidos graxos, $\mathrm{c}_{\mathrm{p}(\mathrm{AG})}{ }^{0} \mathrm{o}$ calor específico do gás ideal, $\mathrm{R}$ a constante universal dos gases, $\mathrm{T}_{\mathrm{r}}$ a temperatura reduzida e $\omega$ o fator acêntrico.

$$
C_{p(\text { estimado })}=C_{p(A G)}+F_{c}
$$

Sendo $F_{c}$ o fator de correção dependente da massa molecular do óleo.

\subsection{Caracterização dos óleos vegetais}

Para avaliar os métodos de previsão de propriedades corretamente, deve-se fazer a escolha de dados consistentes, ou seja, a composição da amostra de óleo estudada deve ser a mais real possível, de forma a garantir que as conclusões sejam eficazes.

Para o óleo de soja, girassol e palma, que foram utilizados como matéria prima neste trabalho, a composição usada em triacilglicerídeos foi à obtida por Cunha e Oliveira (2006), Ma et al. (1999), Dunkle et al. (2012), respectivamente. Esses autores determinaram o perfil do óleo em TGs por cromatografia líquida de alta resolução (HPLC).

Para o óleo da polpa de macaúba, como não foi encontrada na literatura a composição em TGs, DGs e MGs, foram propostas duas metodologias de caracterização. A primeira é uma abordagem em que o óleo é representado por uma mistura de TG-Mistos, seja por (A) cromatografia gasosa de alta resolução ou (B) metodologia computacional proposta por Filho et al. (1995). A segunda abordagem é a caracterização do óleo vegetal sendo uma mistura de ácidos graxos. 


\section{Abordagem 1}

(A) Análise por Cromatografia Gasosa de Alta Resolução (CGAR)

A amostra do óleo da polpa da macaúba foi diluída em tolueno na concentração final de $0,7 \%$. A análise por CGAR foi realizada sem derivatização em coluna capilar de sílica fundida termo estabilizada de TG-5 HT da marca Thermo de 15 metros x $0,25 \mathrm{~mm} \times 0,10$ micrômetros. A análise foi realizada com fluxo de hidrogênio de $1,5 \mathrm{~mL}$ a $50^{\circ} \mathrm{C}$ sob pressão constante. A temperatura inicial da coluna foi de $50^{\circ} \mathrm{C}$, com programação de elevação de temperatura de $15^{\circ} \mathrm{C} /$ minuto até $180^{\circ} \mathrm{C}$, com rampa de $7^{\circ} \mathrm{C} /$ minuto até $230^{\circ} \mathrm{C}$ e até $350^{\circ} \mathrm{C}$ com programação de $10^{\circ} \mathrm{C} / \mathrm{min}$, permanecendo nesta temperatura por mais 25 minutos. $\mathrm{O}$ injetor foi mantido a $320^{\circ} \mathrm{C}$, no modo de divisão de fluxo de 1:50 e foi injetado 1 microlitro de solução. O detector foi mantido a $380^{\circ} \mathrm{C}$.

Para quantificação dos triacilgliceróis foi realizada normalização interna. Para identificação foram utilizados padrões de ácido palmítico, linoleico, monoleína, monopalmitina, dioleína, dipalmitina, tripalmitina e trioleína das marcas SIGMA e NU CHEK, que foram dissolvidos em tolueno PA.

\section{(B) Análise Computacional}

O programa de computador foi montado no Microsoft Excel baseado em equações para calcular a porcentagem molar de triacilglicerídeos de acordo com a distribuição e composição molar de ácidos graxos presentes no óleo vegetal.

Para que a caracterização do óleo da polpa da macaúba fosse realizada era necessário o perfil de ácidos graxos. Assim, foi utilizada a composição mássica obtida por Rio et al. (2016).

Para a construção do perfil do óleo estudado em TGs foram considerados 33 moléculas de triacilgliceróis com 99 radicais de ácidos graxos, o número de cada radical nestas 33 moléculas foi proporcional à percentagem apresentada na Tabela 5. Estes radicais de AGs foram distribuídos aleatoriamente entre as 33 moléculas de TGs.

Assim, as propriedades termofísicas foram estimadas para cada TG misto pela aplicação do método de predição específico e, em seguida, utilizou-se a regra de mistura para estimar a propriedade do óleo de acordo com a composição de triacilgliceróis mistos.

Abordagem 2: A segunda abordagem se baseia na caracterização do óleo vegetal como sendo uma mistura de ácidos graxos. Ou seja, utiliza-se a composição em ácidos graxos do óleo para a determinação das propriedades de interesse. Esta metodologia tem sido a mais utilizada por pesquisadores, por existir maior quantidade de dados experimentais das propriedades dos ácidos graxos puros.

\section{RESULTADOS E DISCUSSÃO}

Para a avaliação completa e precisa dos modelos termodinâmicos, é necessário fazer a comparação com dados experimentais. Para isso, uma vasta pesquisa bibliográfica, acerca desses valores das propriedades estudadas foi realizada em artigos científicos, livros, relatórios de corporações, entre outras. Também foram utilizados banco de dados disponíveis na literatura, tais como: DDB (2019), DIPPR (2019) e NIST (2019).
Todas as comparações entre valores preditos e experimentais foram feitas em termos dos desvios absolutos (DA) conforme a Equação 44, desvios relativos (Equação45) e média dos desvios relativos (MDR) (Equação 46).

$$
\begin{gathered}
\text { Desvios absolutos }(D A)=\left|\mathrm{x}_{\text {exp }, \mathrm{i}}-\mathrm{x}_{\mathrm{calc}, \mathrm{i}}\right| \\
\text { Desvios relativos }(D R)=\frac{\left|\mathrm{x}_{\text {exp,i }}-\mathrm{x}_{\mathrm{calc}, \mathrm{i}}\right|}{X_{\text {exp }, i}} \\
\mathrm{MDR}=\frac{\sum_{i}^{N} \frac{\left|X_{\text {exp }, i}-X_{\text {calc }, i}\right|}{X_{\text {exp }, i}}}{n} \times 100
\end{gathered}
$$

Onde $\mathrm{X}$ é a propriedade a ser avaliada, n é o número de dados experimentais e os subscritos "exp" e "calc" indicam a propriedade experimental e calculada, respectivamente.

\subsection{Estimativa das propriedades termodinâmicas}

As propriedades estimadas para os TGs, DGs, MGs, AGs, ésteres metílicos e etílicos, que serão discutidas a seguir, encontram-se disponíveis nos materiais suplementares.

Temperatura normal de ebulição $\left(\mathrm{T}_{\mathrm{b}}\right)$ : Primeiramente foram estimadas as temperaturas normais de ebulição para triacilgliceróis, cujos dados experimentais foram encontrados na literatura. Os métodos de contribuição de grupos JOBACK e GANI, e o método baseado em fragmentos de ZONG foram avaliados e os dados estimados estão representados na Figura 2.

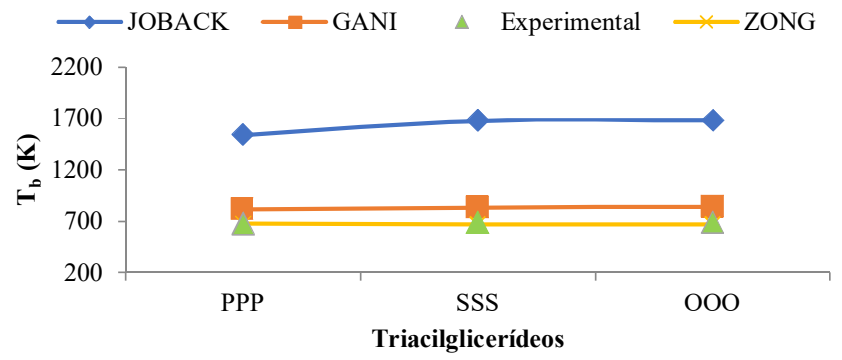

Figura 2- Temperaturas normais de ebulição (K) estimadas comparadas a dados experimentais para triacilgliceróis simples (PPP- tripalmitina, SSS-triestearina e OOOtrioleína) pelos métodos estudados.

Na Figura 2, pode ser observado as $\mathrm{T}_{\mathrm{b}}$ estimadas para três triacilgliceróis simples, PPP, SSS e OOO, comparados a dados experimentais obtidos em Goodrum e Geller (2002) e Santander et al. (2012). Os resultados mostram que os valores de $\mathrm{T}_{\mathrm{b}}$ calculados pelo método de JOBACK possuem desvios altos (MDR $>100 \%$ ). A média dos desvios relativos mais baixa foi atingida quando o método $\mathrm{ZONG}$ foi utilizado ( $\mathrm{MDR}=1,9 \%$ ), seguida de $21 \%$ para GANI. Assim, o método de ZONG foi empregado neste trabalho para o cálculo das $\mathrm{T}_{\mathrm{b}}$ para os óleos vegetais, na abordagem em TGs.

Como não foram encontrados na literatura dados experimentais dos MGs e DGs, o método adotado para a determinação das $T_{b}$ foi o método de $Z O N G$, o qual observou menores desvios para os TGs analisados, conforme mostrado anteriormente.

Os desvios relativos da estimativa de $\mathrm{T}_{\mathrm{b}}$ para os ácidos graxos de acordo com JOBACK e GANI, são apresentados na Figura 3. Os dados experimentais utilizados encontram-se em NIST (2019) e DDB (2019). Vale ressaltar que não é possível 
aplicar a metodologia baseada em fragmentos proposta por Zong et al. (2010a) para esses compostos.

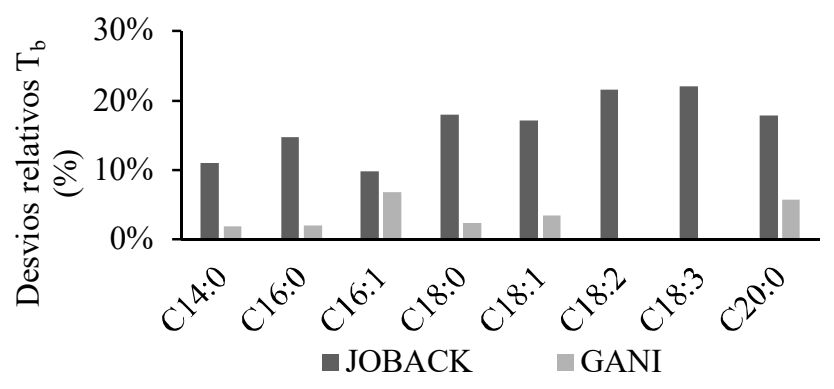

Figura 3 - Desvios relativos (\%) para a temperatura normal de ebulição para ácidos graxos nos métodos de JOBACK e GANI.

Pela análise da Figura 3, observa-se que o maior desvio do método de GANI ocorreu no ácido graxo insaturado C16:1. A média dos desvios relativos (MDR) encontrada para os ácidos graxos saturados foi $15,4 \%$ para o método de JOBACK e $2,9 \%$ para GANI. Por outro lado, os AGs insaturados deram MDR iguais $17,6 \%$ e $2,5 \%$ para os mesmos métodos, respectivamente. Esta análise mostra a concordância da estimativa da $T_{b}$ pelo método de GANI tanto para os AGs saturados e insaturados.

Para reforçar a análise anterior, uma curva da $\mathrm{T}_{\mathrm{b}}$ em função da massa molar dos AGs saturados e insaturados foi construída (Figura 4). Nesta figura, os pontos de ebulição dos ácidos graxos saturados (linha contínua) mostram uma característica quase linear. Além disso, pode-se notar a proximidade dos dados experimentais com os valores estimados pelo método de GANI.

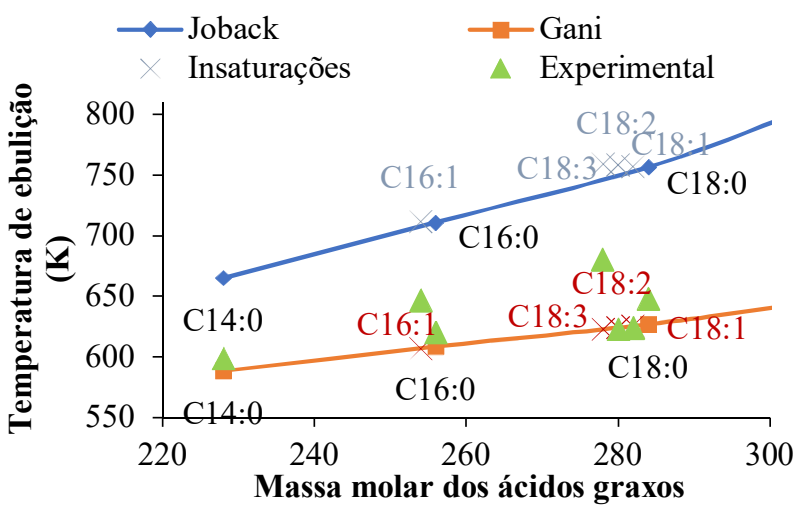

Figura 4- Temperaturas normais de ebulição (K) estimadas em função da massa molar para ácidos graxos pelo método de Joback e Gani em comparação com dados experimentais.

É importante ressaltar que, em um estudo de simulação de produção de biodiesel, um erro no valor de $\mathrm{T}_{\mathrm{b}}$ implicará na previsão errônea para outras propriedades, como por exemplo, as propriedades críticas. Este fato leva a resultados errados quando as propriedades, tais como, viscosidade e densidade, forem calculadas usando equações e correlações incorporadas em simuladores comerciais.

Propriedades críticas $\left(\mathrm{P}_{\mathrm{c}}, \mathrm{V}_{\mathrm{c}}, \mathrm{T}_{\mathrm{c}}\right.$ e $\left.\mathrm{Z}_{\mathrm{c}}\right)$ : As propriedades críticas foram estimadas para os TGs, DGs e MGs para os métodos de AMBROSE, JOBACK, GANI e LYDERSEN.

É importante mencionar que foram encontrados apenas dados a temperatura crítica de alguns AGs (NIST, 2019). Não estavam disponíveis valores para os TGs, DGs e MGs. No entanto, foram considerados suficientes para apontar o método mais preciso.

As médias dos desvios relativos (MDR) obtidas para as temperaturas críticas preditas para os AGs comparadas aos dados experimentais são $3,31 \%, 0,52 \%, 3,57 \%$ e $2,40 \%$, respectivamente, para os métodos JOBACK, GANI, LYDERSEN e AMBROSE.

Nota-se, que o método de GANI apresentou média dos desvios relativos menores, para os ácidos graxos $(\mathrm{MDR}=0,52 \%)$. Acrescido a isso, esse método é o único dentre os avaliados que necessita, como entrada, apenas a estrutura molecular (ASPEN TECH, 2014). Assim, esta metodologia foi escolhida para a predição das propriedades críticas dos componentes dos óleos vegetais.

Sales-Cruz et al. (2010) estimaram propriedades críticas para vários compostos, envolvidos na produção de biodiesel. Foram analisados ácidos graxos, triacilgliceróis e ésteres metílicos. Os autores compararam os dados obtidos com dados experimentais e, apontaram GANI como o método mais preciso, diante dos outros dois estudados, Joback-Reid (1987) e Marrero-Gani (2001).

Por outro lado, quando a estimativa das propriedades críticas é realizada por métodos que necessitam da $T_{b}$, observouse uma variabilidade nos métodos indicados como mais precisos. An et al. (2013) estimaram as propriedades críticas através dos métodos de Ambrose et al. (1980) e Joback e Reid (1987). Segundo os autores, método de Ambrose apresentou os menores desvios em relação aos dados disponíveis no banco de dados DIPPR para temperatura e pressão críticas. Anand et al. (2011) avaliaram a temperatura crítica pelos os métodos de Fedors (1979 e 1982), Joback e Reid (1994) e, Klincewicz e Lyndersen (REID et al., 1987). Comparando os resultados com valores preditos no banco de dados NIST, os autores selecionaram os grupos de Lydersen e Joback e ressaltam o sucesso destes métodos por considerarem o valor experimental da temperatura normal de ebulição na predição da temperatura crítica.

Fator acêntrico $(\omega)$ : Os dados estimados pela regra de Pitzer (1955) e Kesler-Lee (1976) apresentaram resultados semelhantes, conforme pode ser visualizado na Tabela 2.

Tabela 2 - Dados estimados do fator acêntrico para trilinoleína (LLL), dilinoleína (LL) e monolinoleína (L)

\begin{tabular}{l|cc} 
Componentes & Pitzer & Kesler-Lee \\
\hline LLL & $-0,6524$ & $-0,6343$ \\
LL & $-0,3183$ & $-0,3183$ \\
L & 1,0146 & 1,0692 \\
\hline
\end{tabular}

Neste trabalho, adotou-se a regra de Pitzer para a determinação dos fatores acêntricos de todos os compostos, por ser o método mais utilizado segundo Reid et al. (1987).

Entalpia de formação e energia de Gibbs no estado padrão $\left(\mathrm{H}_{\mathrm{f}}^{0}\right.$ e $\left.\mathrm{G}_{\mathrm{f}}^{0}\right)$ : Inicialmente, os dados da entalpia de formação foram avaliados para o TG (trioleína-OOO), DG (dioleína-OO) e MG (monoleína- O) em comparação com dados disponíveis na literatura (DIPPR, 2019; Vatani et al., 2007).

A Tabela 3 mostra os desvios absolutos obtidos para cada 
método utilizado na estimativa desta propriedade. Nota-se que, os métodos avaliados apresentaram desvios médios relativos abaixo de 3\%. A menor MDR foi observada quando GANI $(0,75 \%)$ foi utilizado, seguido de JOBACK $(0,95 \%)$. Entretanto, o método de GANI exige como entrada a temperatura normal de ebulição. Assim para evitar o erro associado a uma medida previamente estimada, o método escolhido neste trabalho foi JOBACK. Este exige como entrada apenas a estrutura molecular dos compostos. Da mesma forma que a entalpia de formação, as energias de Gibbs foram calculadas por JOBACK para os TGs, DGs e MGs.

Tabela 3 - Desvios absolutos e média dos desvios relativos (MDR) da entalpia padrão de formação de TG, DG e MG comparados a dados da literatura.

\begin{tabular}{|c|c|c|c|c|}
\hline & \multirow{2}{*}{$\begin{array}{c}\text { DIPPR } \\
801(\mathrm{~kJ} / \mathrm{mol})\end{array}$} & \multicolumn{3}{|c|}{ Desvios absolutos ( $\mathrm{kJ} / \mathrm{mol})$} \\
\hline & & JOBACK & GANI & BENSON \\
\hline$O O O$ & -2161 & 14,415 & 1,078 & 35,053 \\
\hline$O O$ & -1670 & 14,844 & 16,090 & 44,916 \\
\hline$O$ & -1175 & 15,345 & 14,681 & 45,660 \\
\hline & $\operatorname{MDR}(\%)$ & 0,954 & 0,754 & 2,733 \\
\hline
\end{tabular}

Para os AGs, foram obtidos na literatura apenas dados da entalpia de formação do ácido esteárico (-884,7 kJ/mol) (NIST, 2019). Os desvios absolutos (DA) encontrados para os métodos de JOBACK, GANI e BENSON, respectivamente foram de $119,58,121,14$ e $389,22 \mathrm{~kJ} / \mathrm{mol}$. Portanto, o primeiro método foi escolhido para predizer a energia de formação para os ácidos graxos.

Para a energia de Gibbs o método escolhido para os ácidos graxos foi BENSON (Reid et al.,1987). Segundo Aspen Plus (2012), este é mais preciso na estimativa dessa propriedade em comparação com os métodos de GANI e JOBACK, por considerar os efeitos dos átomos vizinhos nos cálculos.

Pressão de vapor $\left(\mathrm{P}_{\mathrm{VAP}}\right)$ : A pressão de vapor foi, inicialmente, avaliada para TGs simples pelo método de contribuição de grupos (CERIANI et al.; 2013) e método baseado em fragmentos (ZONG et al., 2010a; Zong et al., 2010b). A Figura 5 compara os dados estimados da $\mathrm{P}_{\text {vap }}$ comparados a dados experimentais para a triestearina (SSS) e tripalmitica (PPP), obtidos em Perry et al. (1949).

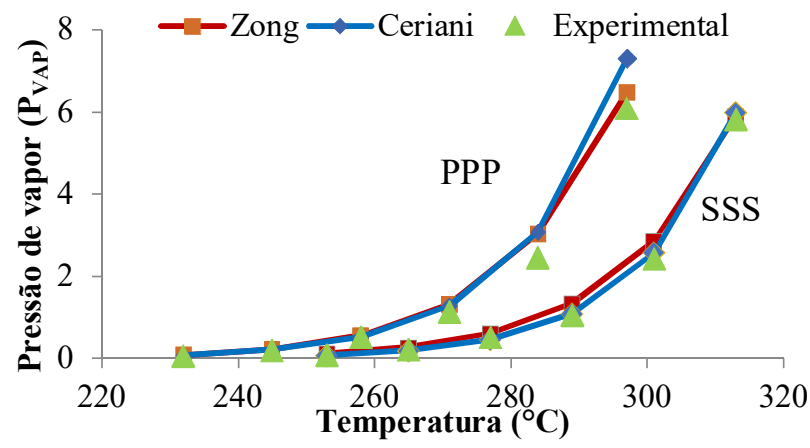

Figura 5 - Dados experimentais e estimados de pressão de vapor ( $\mathrm{Pa}$ ) para os métodos estudados para tripalmitina (PPP) e triestearina (SSS).

Os métodos de Ceriani et al. (2013) e Zong et al. (2010a e 2010b) mostraram previsões similares para os TGs conforme mostrado na Figura 5. As médias dos desvios das pressões de vapor estimadas, no intervalo de temperatura de 230 a $313^{\circ} \mathrm{C}$, para os triacilgliceróis simples tripalmitina (PPP) e triestearina
(SSS) foram respectivamente, 15,01 e 20,04 para ZONG e 6,63 e 19,58 para CERIANI. Isso resultou em MDRs para o método de ZONG de $17,52 \%$ e para a CERIANI $13,11 \%$. Pela análise das MDRs, o método de Ceriani et al. (2013) apresentou maior precisão, então, foi apontado para a estimativa da pressão de vapor para os TGs.

Além disso, segundo Ceriani et al. (2013), o método proposto é capaz de representar qualquer número de compostos graxos e hidrocarbonetos com o menor número de parâmetros e pode-se extrapolar a temperatura (ponto crítico), ao descrever corretamente as pressões de vapor e calores de vaporização.

Entalpia de vaporização (HAP $)$ : A Tabela 4 mostra os valores estimados para o calor de vaporização comparados a dados obtidos no banco de dados NIST (2019).

Tabela 4 - Desvios relativos (DR) e média dos desvios relativos (MDR) da entalpia de vaporização

\begin{tabular}{|c|c|c|c|c|c|}
\hline \multicolumn{3}{|c|}{ Temper } & \multicolumn{3}{|c|}{ DR (\%) } \\
\hline & $\begin{array}{c}\text { atura } \\
\left({ }^{\circ} \mathrm{C}\right)\end{array}$ & $\begin{array}{c}\text { Exp. } \\
(k J / m o l)^{I}\end{array}$ & $\begin{array}{l}\text { Ceriani } \\
(2013)\end{array}$ & $\begin{array}{l}\text { Pitzer } \\
(1995)\end{array}$ & $\begin{array}{c}\text { Basaro } \\
\text { va } \\
(1995)\end{array}$ \\
\hline SSS & $247-314$ & 164,63 & 13,84 & 27,74 & 2,89 \\
\hline PPP & $232-300$ & 158,53 & 6,27 & 23,36 & 2,47 \\
\hline & & $\operatorname{MDR}(\%)$ & 10,05 & 25,55 & 2,68 \\
\hline
\end{tabular}

Pela a análise da Tabela 4, a menor MDR foi observada para o método de Basarova (MDR $=2,68 \%$ ), seguido por Ceriani $(\mathrm{MDR}=10,05 \%)$ e Pitzer $(\mathrm{MDR}=25,55 \%)$. Vale ressaltar que, para os métodos de Pitzer (1995) e Basarova e Svoboda (1995), é necessário informar as temperaturas e pressões críticas, e os fatores acêntricos dos TGs. Assim, estes métodos tornam-se menos convenientes de serem utilizados, uma vez que são necessários usar dados estimados previamente, ou seja, pode provocar erros cumulativos.

Su et al. (2011) indicaram o método de Basarova e Svoboda (1995) para prever valores da entalpia de vaporização quando se utilizou o método de Ceriani e Meirelles (2004). Por sua vez, Ceriani et al. (2013) corrigiram as limitações de temperatura do método anteriormente proposto (CERIANI e MEIRELLES, 2004). Então, o método adotado neste trabalho para prever a $\Delta \mathrm{H}_{\mathrm{va}} \mathrm{p}$ dos TGs, DGs e MGs foi Ceriani et al. (2013).

Densidade $(\rho)$ : A Tabela 5 compara as previsões de densidades por Halvrosen et al. (1993), Zong et al. (2010a e 2010b) e Ihmels Gmehling (2003) a dados experimentais de TGs simples (EITEMAN e GOODRUM, 1994; PHILLIPS e MATTAMAL, 1978 e SUM et al., 2003).

Tabela 5 - Média dos desvios relativos (MDR) das densidades estimadas para TGs simples nas temperaturas 80-100 ${ }^{\circ} \mathrm{C}$.

\begin{tabular}{c|ccc|}
\hline \multirow{2}{*}{ Componentes } & \multicolumn{3}{c|}{ MDR (\%) } \\
HALVROSEN & ZONG & IHMELS \\
PSS & 0,3 & 0,2 & 1,1 \\
OOO & 3,6 & 4,0 & 2,7 \\
LLL & 2,8 & 2,1 & 1,5 \\
\hline
\end{tabular}

Todos os três métodos avaliados garantem uma precisão ao prever a densidade para os TGs no intervalo de temperatura de $80^{\circ} \mathrm{C}$ a $100^{\circ} \mathrm{C}$. As médias dos desvios encontrados foram 
baixas, não ultrapassando $4,0 \%$.

O método de Halvorsen et al. (1993) não possui fatores de correção adequados para os DGs e MGs da equação Rackett para estimar a densidade de óleos vegetais. Além disso, o método de contribuição de grupos de Ihmels e Gmehling (2003) não é particularmente concebido para previsões de densidade de compostos lipídicos. Ou seja, não há um grupo específico para descrever a porção de glicerol em TGs, DGs, MGs e óleos. Por isso, grupos $\mathrm{CH}_{2}, \mathrm{CH}, \mathrm{CH}_{2} \mathrm{OH}$, e $\mathrm{CHOH}$ devem ser utilizados como substitutos ao descrever a porção do glicerol. Para melhorar a precisão do método, deve-se desenvolver os dados para o grupo glicerol.

Considerando que o método de Zong et al. (2010a e 2010b) é baseado em fragmentos, geralmente, mais preciso que os métodos de contribuição de grupos, e ainda, calculando a $\mathrm{MDR}_{\text {total }}$ para os métodos avaliados, a ordem crescente observada das MDRs totais das densidades é ZONG $(1,6 \%)<$ IHMELS $(1,7 \%)<$ HALVROSEN $(2,0 \%)$. Contudo, o método de ZONG foi escolhido para prever as densidades de TGs, DGs e MGs

A Figura 6 ilustra a boa concordância do método de Ihmels e Gmehling (2003) para o cálculo da densidade dos ácidos graxos, éteres metílicos e etílicos. Para essa comparação foram utilizados dados experimentais de Noureddini et al. (1992) e Pratas et al. (2010). Nesta, as linhas contínuas são dados estimados e os pontos são valores experimentais, cores iguais significam o mesmo ácido graxo.

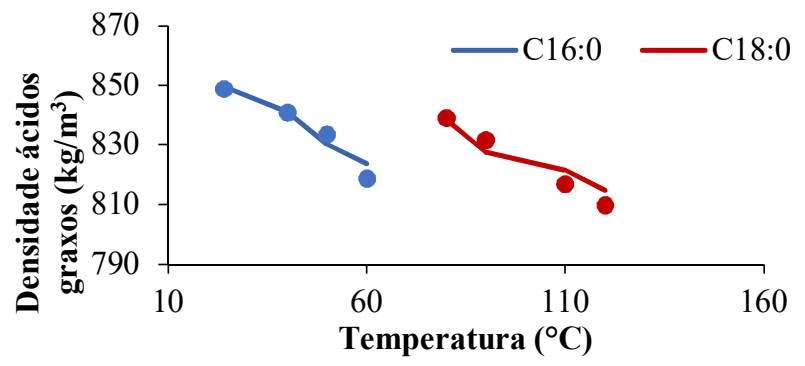

Figura 6 - Densidades $\left(\mathrm{kg} / \mathrm{m}^{3}\right)$ estimadas em função da temperatura de AGs, comparados a dados experimentais. As linhas contínuas representam os dados estimados e os pontos valores experimentais obtidos da literatura.

A MDR (\%) obtida para os ácidos graxos para o método de Ihmels e Gmehling (2003) foram baixas (0,30\%). Mostrando que o método é preciso ao estimar a densidade para estes compostos.

Capacidade calorífica (cp): Os desvios das capacidades caloríficas estimadas comparados a dados experimentais de Philips e Mattamal (1976) e Morad et al. (2000) podem ser vistos na Tabela 12. Os dados foram estimados no intervalo de temperatura de $60-180^{\circ} \mathrm{C}$.

Os resultados obtidos, conforme apresentado na Tabela 12, mostraram que o método Zong et al. (2010a) têm maior precisão que de Ceriani et al. (2009) e Morad et al (2000). Neste caso específico, a média dos desvios relativos totais para o método de ZONG foi menor $0,88 \%$, enquanto para o método Ceriani et al. (2009) e Morad et al. (2000) essas foram de 3,26\% e $1,51 \%$, respectivamente. Ressalta-se que o método de Morad et al. (2000) é aplicável somente aos TGs.

Tabela 6 - Média dos desvios relativos (MDR) das capacidades caloríficas estimadas para TGs simples

\begin{tabular}{c|ccc} 
Componentes & \multicolumn{3}{c}{ MDR (\%) } \\
\cline { 2 - 4 } & MORAD & ZONG & CERIANI \\
PPP & 2,74 & 1,24 & 2,21 \\
SSS & 1,10 & 1,24 & 1,33 \\
OOO & 0,68 & 0,15 & 6,25 \\
\hline MDR $_{\text {total }}(\mathbf{\%})$ & $\mathbf{1 , 5 1}$ & $\mathbf{0 , 8 8}$ & $\mathbf{3 , 2 6}$ \\
\hline
\end{tabular}

Portanto, adotou-se a metodologia proposta por Zong et al. (2010a) para os cálculos desta propriedade para os TGs, DGs e MGs.

\subsection{Caracterização dos óleos}

O óleo da polpa da macaúba foi caracterizado pela sua composição de duas maneiras, conforme citado no item 2.2 deste capítulo.

\section{Abordagem 1:}

(A) Análise por Cromatografia Gasosa de Alta Resolução (CGAR)

Os resultados da análise cromatográfica podem ser observados na Tabela 7.

Tabela 7 - Composição do óleo da polpa da Macaúba em TGs obtido por CGAR

\begin{tabular}{|c|ccc|}
\multicolumn{1}{c}{ Amostra } & \multicolumn{3}{c|}{ Óleo de polpa de macaúba } \\
\hline Triacilglicerol & Rep. 1 & Rep. 2 & $\begin{array}{c}\text { Exemplos de } \\
\text { TGs possíveis }\end{array}$ \\
\hline C48 & 0,77924 & 0,80121 & PPP \\
C50 & 9,96296 & 9,79972 & PPS, PPO, PPL, \\
& & & PPLn \\
C52 & 42,1804 & 41,928 & SSP \\
C54 & 47,077 & 47,471 & SSS, OOO, LLL \\
\hline
\end{tabular}

Nota: Não há diferenciação entre C18:0, C18:1 e C18:2

Como nesta técnica foi utilizada uma coluna capilar não polarizada, a análise é simplificada, pois os TGs são separados em grupos tendo o mesmo número de átomos de carbono, não sendo possível a determinação das insaturações.

Uma separação mais refinada dos TGs ocorre quando se é utilizada uma coluna polarizada na análise cromatográfica. Assim, em uma coluna polarizada, a separação de TGs, tais como POO e PLS, que são TGs com o mesmo número de carbonos e grau de insaturação, mas com polaridade diferente, pode ser alcançada. Segundo Filho et al. (1995) em ambas as colunas, não polares e polarizadas, não há discriminação entre triacilglicerídeos isoméricos como POO e OPO.

\section{(B) Análise Computacional}

$\mathrm{Na}$ Tabela 8 são mostradas as proporções dos triacilgliceróis através da composição dos ácidos graxos obtidos do programa computacional criado neste trabalho conforme metodologia citada por Filho et al. (1995).

A identificação dos triacilgliceróis por grupos com diferentes números de átomos de carbono e por diferentes combinações de ácidos graxos saturados e insaturados foram feitas comparando os dados percentuais obtidos da CGAR com os fornecidos pelo programa criado no computador. Os dados na Tabela 9 mostram que as proporções de cada componente calculada pelo computador (PC), são próximas da obtida média 
por análise cromatográfica (CGAR).

Tabela 8 - Caracterização estimada do óleo da polpa da macaúba em TGs

\begin{tabular}{c|ccc|cc} 
Grupos & TGs & $\boldsymbol{\%} \boldsymbol{m} / \boldsymbol{m}$ & Grupos & TGs & $\boldsymbol{\%} \boldsymbol{m} / \boldsymbol{m}$ \\
\hline C50:1 & POP & 8,0 & C52:4 & PLnO & 4,0 \\
C50:1 & PPoS & 2,0 & C54:2 & SOO & 4,0 \\
C52:2 & POO & 28,0 & C54:3 & OOO & 26,0 \\
C52:3 & OPoO & 4,0 & C54:4 & OOL & 14,0 \\
C52:3 & PLO & 2,0 & C54:5 & OLL & 2,0 \\
C52:4 & LOPo & 6,0 & & & \\
\hline
\end{tabular}

Tabela 9 - Composição do óleo da polpa da macaúba em triacilgliceróis por CGAR e pelo uso de um programa elaborado no computador (PC)

\begin{tabular}{|c|c|c|}
\hline \multirow{2}{*}{$\begin{array}{l}\text { Número de } \\
\text { Carbonos }\end{array}$} & \multicolumn{2}{|c|}{ Porcentagem da composição } \\
\hline & PC & CGAR \\
\hline $\mathrm{C} 48$ & 0,00 & 0,79 \\
\hline C50 & 10,00 & 9,88 \\
\hline C52 & 44,00 & 42,05 \\
\hline $\mathrm{C} 54$ & 46,00 & 47,27 \\
\hline
\end{tabular}

Portanto, a lista dos triacilgliceróis apresentada na Tabela 8 , obtida pelo programa de computador, é usada como composição do óleo da polpa da macaúba para o presente trabalho. Isso porque a abordagem em triacilgliceróis mistos aproxima-se mais da realidade, além dos resultados da análise cromatográfica mostrarem que o programa utilizado para a distribuição dos TGs mistos atende aos dados obtidos experimentalmente.

\subsection{Seleção do método de caracterização do óleo}

Por não ter sido encontrada a composição em TGs do óleo da polpa da macaúba, duas metodologias foram avaliadas. A escolha do método de caracterização adequado é importante na previsão das propriedades termofísicas. Alguns critérios a serem considerados na adoção do método são: a facilidade de uso, caracterização rigorosa, e previsões satisfatórias. A Tabela 8 e Rio et al. (2016) mostraram a composição do óleo da macaúba em termos de TGs e ácidos graxos, respectivamente.

Foram realizadas previsões de algumas propriedades para comparar as duas abordagens para caraterização do óleo. A Tabela 10 mostra previsões da densidade e capacidade calorífica do óleo da polpa da macaúba para a abordagem em TGs mistos e em ácidos graxos.

Tabela 10 - Médias dos desvios relativos (MDR) das densidades e capacidades caloríficas pelas duas abordagens de caracterização do óleo da polpa da macaúba

\begin{tabular}{c|lcc}
\hline \multicolumn{1}{c}{} & \multicolumn{1}{c}{ Método } & $\begin{array}{c}\text { TG- } \\
\text { Mistos }\end{array}$ & $\begin{array}{c}\text { Acidos } \\
\text { graxos }\end{array}$ \\
\cline { 2 - 4 } Densidade & Halvorsen et al. $(1993)$ & 0,17 & 0,16 \\
& Zong et al. $(2010 \mathrm{a})$ & 0,97 & $\mathrm{ND}$ \\
& Ihmels et al. $(2003)$ & 1,79 & 1,83 \\
\hline \multirow{2}{*}{ Capacidade } & Morad et al. $(2000)$ & 2,17 & 2,50 \\
calorífica & Zong et al. $(2010 \mathrm{a})$ & 2,20 & $\mathrm{ND}$ \\
& Ceriani et al. $(2009)$ & 0,56 & 0,47 \\
\hline
\end{tabular}

Pela análise da Tabela 10, pode-se observar que as duas abordagens tiveram estimativas semelhantes, com média dos desvios relativos pequenas. No entanto, recomenda-se a utilização da caracterização dos óleos em TGs mistos, por ser mais próxima ao real.

\subsection{Propriedades dos óleos vegetais}

Sabe-se que o óleo vegetal é uma mistura de vários TGs, DGs, MGs e AGs livres. Adotou-se, neste trabalho, que os óleos vegetais eram compostos apenas de TGs e DGs. Para a determinação das propriedades dos óleos vegetais foram utilizadas suas composições apresentadas anteriormente e as propriedades preditas dos compostos presentes na mistura, utilizando a regra de mistura ideal (equação 47).

$$
P_{\text {mistura }}=\sum_{i=1}^{n} x_{i} P_{\text {puro }, i}
$$

Os métodos adotados para predizer as propriedades termofísicas dos TGs, DGs e DGs encontram-se resumidos na Tabela 11.

Tabela 11 - Métodos adotados para predizer as propriedades termofísicas dos óleosTGs, DGs e MGs

\begin{tabular}{|c|c|}
\hline Propriedades & Métodos \\
\hline$T_{b}$ & Zong et al. (2010a e 2010b) \\
\hline$T_{c}, P_{c}$ e $V_{c}$ & Constantinou e Gani (1994) \\
\hline Fator acêntrico $(\omega)$ & Pitzer (1995) \\
\hline Entalpia de formação $\left(H_{f 0}\right)$ & Joback e Reid (1987) \\
\hline Energia de Gibbs $\left(G_{f 0}\right)$ & Joback e Reid (1987) \\
\hline $\mathrm{P}_{\mathrm{VAP}}$ & Ceriani et al (2013) \\
\hline $\mathrm{H}_{\text {vap }}$ & Ceriani et al (2013) \\
\hline$\rho$ & Zong et al. $\left(2010^{\mathrm{a}}\right.$ e $\left.2010^{\mathrm{b}}\right)$ \\
\hline$c_{p}{ }^{L}$ & Zong et al. $\left(2010^{\mathrm{a}}\right.$ e $\left.2010^{\mathrm{b}}\right)$ \\
\hline
\end{tabular}

Então, as propriedades estimadas para os óleos vegetais foram comparadas com dados experimentais disponíveis na literatura. A representação das propriedades termofísicas dependentes da temperatura, tais como entalpia de vaporização $\left(\mathrm{H}_{\mathrm{vap}}\right)$, capacidade calorífica $\left(\mathrm{c}_{\mathrm{pL}}\right)$, densidade $(\rho)$ e pressão de vapor ( $p_{\text {vap }}$ ) é mostrada na Figura 7(a), Figura 7(b), Figura 7(c) e Figura 7(d), respectivamente.

Pela análise da Figura 7 (a), observa-se que a entalpia de vaporização dos óleos vegetais diminui com o aumento da temperatura. Hammond et al. (2005) determinaram o calor de vaporização para o óleo de soja, $\mathrm{H}_{\mathrm{vap}}=184,9 \mathrm{~kJ} / \mathrm{mol}$. O valor predito para o óleo de soja, neste trabalho, foi no intervalo de 216 a $174 \mathrm{~kJ} / \mathrm{mol}$, mostrando que há concordância do dado predito com o experimental. Pela análise da Figura 7 (b), podese observar que as capacidades caloríficas dos óleos vegetais analisados aumentaram linearmente com o aumento da temperatura desde 20 a $80^{\circ} \mathrm{C}$. Comportamento semelhante foi observado por Fasina e Colley (2008).

Ceriani et al. (2008) determinaram a densidade e a viscosidade para óleo de babaçu, buriti, castanha do Pará, macadâmia e semente de uva. As densidades encontradas foram de 912 a $876 \mathrm{~kg} / \mathrm{m}^{3}$, no intervalo de temperatura de $20-70^{\circ} \mathrm{C}$. Strecker et al. (1996) determinaram a densidade do óleo de palma, $\rho=888 \mathrm{~kg} / \mathrm{m}^{3}$ a $50^{\circ} \mathrm{C}$, próximo ao valor encontrado neste trabalho $\left(880 \mathrm{~kg} / \mathrm{m}^{3}\right)$. Para o óleo de girassol, esses autores encontraram densidade no intervalo de 880 a $945 \mathrm{~kg} / \mathrm{m}^{3}$ na faixa de temperatura de -20 a $80^{\circ} \mathrm{C}$. Valores similares foram encontrados, conforme é apresentado na Figura 7 (c). 


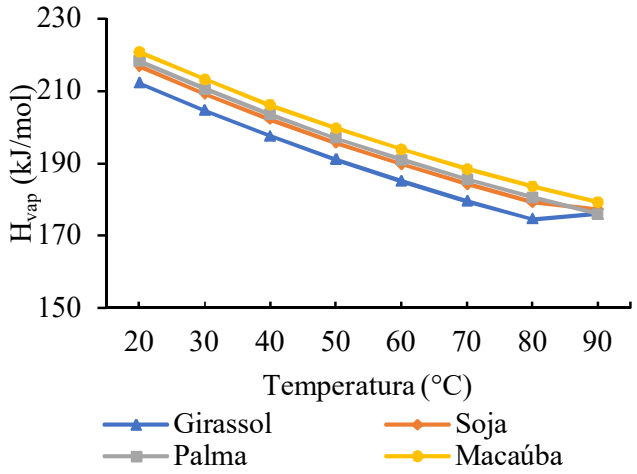

(a)

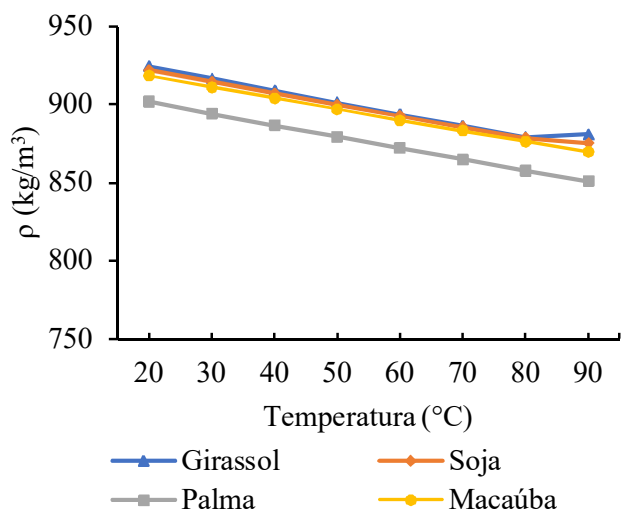

(c)

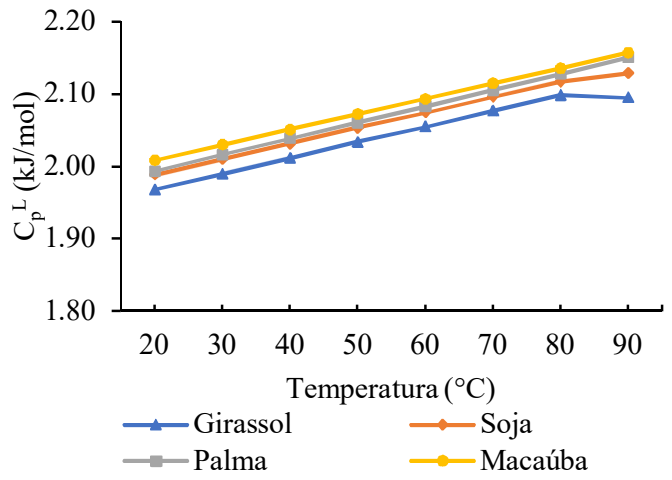

(b)

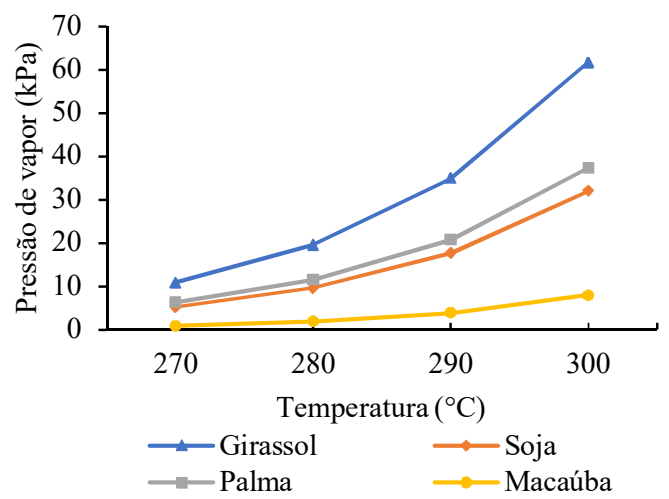

(d)

Figura 7 - Propriedades termofísicas dependentes da temperatura (a) Entalpia de vaporização (b) Capacidade calorífica (c) Densidade e (d) Pressão de vapor estimadas para o óleo de girassol (A), palma (ם), soja (४) e polpa de macaúba (O).

Rodrigues (2007) determinou para o óleo da polpa da macaúba densidades no intervalo de 918,5 a $925,3 \mathrm{~kg} / \mathrm{m}^{3}$, mostrando novamente que as estimativas calculadas, neste trabalho, atingiram boa precisão.

Murata et al. (1993) mediram a pressão de vapor de vários óleos vegetais. Os autores encontraram pressões de vapor para o óleo de soja no intervalo de $2,6 \mathrm{kPa}$ a $73,5 \mathrm{kPa}$ variando a temperatura de $250-330^{\circ} \mathrm{C}$. Foram observados valores similares, conforme pode ser visualizado na Figura 7 (d).

A Tabela 12 mostra o restante das propriedades termofísicas que foram estimadas para os diferentes óleos vegetais em estudo, as quais poderão ser utilizadas como dados de entrada para projetos de simulações.

Enfim, os valores preditos neste trabalho encontram-se em faixas aceitáveis quando comparados com dados experimentais de óleos vegetais similares. A diferença na composição dos óleos, principalmente no número de insaturações, provocam alterações nas propriedades termodinâmicas, conforme foi visto nas análises anteriores.
Tabela 12 - Propriedades estimadas para o óleo da polpa da macaúba, soja, girassol e palma pelos métodos adotados neste trabalho

\begin{tabular}{|c|c|c|c|c|}
\hline Propriedades & Girassol & Soja & Palma & Macaúba \\
\hline$T_{b}(K)$ & 583,34 & 662,40 & 615,54 & 739,08 \\
\hline$T_{c}(K)$ & 836,34 & 950,30 & 879,71 & 1051,57 \\
\hline$P_{c}\left(N / m^{2}\right)$ & $2,95 E+05$ & $3,36 \mathrm{E}+05$ & $3,17 \mathrm{E}+05$ & $3,86 \mathrm{E}+05$ \\
\hline$V_{c}\left(\mathrm{~m}^{3} / \mathrm{kmol}\right)$ & 2,80 & 3,18 & 2,91 & 3,48 \\
\hline$Z_{C}$ & 0,04 & 0,05 & 0,03 & 0,03 \\
\hline$H_{f}(\mathrm{~J} / \mathrm{kmol})$ & $-1,40 \mathrm{E}+09$ & $-1,76 \mathrm{E}+09$ & $-1,84 \mathrm{E}+09$ & $-2,09 \mathrm{E}+09$ \\
\hline$G_{f}(\mathrm{~J} / \mathrm{kmol})$ & $-6,95 E+07$ & $-2,16 \mathrm{E}+08$ & $-3,99 \mathrm{E}+08$ & $-3,73 E+08$ \\
\hline$\omega$ & $-0,59$ & $-0,66$ & $-0,62$ & $-0,75$ \\
\hline
\end{tabular}

\section{CONCLUSÃO}

Este trabalho mostrou que a modelagem de propriedades de TGs, DGs, MGs e ácidos graxos está intimamente ligada à estimativa de parâmetros base, tais como temperatura normal de ebulição; temperatura, pressão e volume críticos e fator acêntrico. Além disso, mostrou-se que o uso de dados 
experimentais para validação do método deve ser realizado com cautela tendo em vista como que a natureza de muitos dados disponíveis é predita.

Modelos de predição de propriedades como a contribuição de grupos e abordagem por fragmentos foram avaliados para determinar o ponto normal de ebulição, propriedades críticas, a pressão de vapor, densidade do líquido, capacidade calorífica e entalpia de vaporização de ácidos graxos, triacilgliceróis, diacilgliceróis e monoacilgliceróis. Pôde-se chegar a valores similares aos experimentais das propriedades com a aplicação destes métodos.

A estimativa das propriedades termofísicas dos óleos vegetais é de suma importância quando se deseja avaliar processos de produção de biocombustíveis pela técnica de simulação. Isso porque, os dados experimentais dessas matérias primas são escassos na literatura. Portanto, é necessário um banco de dados com valores próximos ao real para que o uso de simuladores tenha previsões corretas. Assim, neste trabalho foi criado um banco de dados que poderá ser utilizado como parâmetros de entrada nos projetos de simulação.

\section{REFERÊNCIAS}

AMBROSE, D. Correlation and Estimation of Vapour-Liquid Critical Properties. I Critical Temperatures of Organic Compounds. National Physical Laboratory, Teddington, NPL Rep. Chem. n. 92, 1980.

AN, H.; YANG, W. M.; MAGHBOULI, A.; CHOU, S. K.; CHUA, K. J. Detailed physical properties prediction of pure methyl esters for biodiesel combustion modeling. Applied Energy, 102, 647-656, 2013.

ANAND, K.; SHARMA, R. P.; MEHTA, P. S. A comprehensive approach for estimating thermo-physical properties of biodiesel fuels. Applied Thermal Engineering, n. 31, p.235-242, 2011.

ASPEN PLUS. Aspen Plus: User Guide. Aspen plus v8.0. 936 p. Aspen Technology, Inc., Cambridge (MA), 2012.

ASPEN TECH. Disponível em: $<$ http://www.aspentech.com/products/aspen-plus.aspx $>$. Acesso em: 30 abr 2014.

ATABANI, A. E.; SILITONGA, A. S.; BADRUDDIN, I. A.; MAHLIA, T. M. I.; MASJUKI, H. H.; MEKHILEF, S. A Comprehensive Review on Biodiesel as an Alternative Energy Resource and Its Characteristics. Renewable Sustainable Energy Rev. 2012, 16 (4), 2070ATKINS, P.W; PAULA, J. Físico-química. Vol. 1. LTC, $9^{\mathrm{a}}$ ed., 2012.

BASAROVA, P.; SVOBODA, V. Prediction of the Enthalpy of Vaporization by the Group Contribution Method. Fluid Phase Equilib. 105, 27-47, 1995.

BENSON, S. W.; CRUICKSHANK, F. R.; GOLDEN, D. M.; HAUGEN, G. R.; O’NEAL, H. E.; RODGERS, A. S.; SHAW, R.; WALSH, R. Additivity rules for the estimation of thermochemical properties. Chemical Reviews, n. 3, v. 69, 279-324, 1969.

CERIANI, R.; GANI, R.; LIU, Y. A. Prediction of vapor pressure and heats of vaporization of edible oil/fat compounds by group contribution. Fluid Phase Equilibria, n. 337, p.53-59, 2013.
CERIANI, R.; GANI, R.; MEIRELLES, A. J. A. Prediction of heat capacities and heats of vaporization of organic liquids by group contribution methods. Fluid Phase Equilibria, n.283, p.49-55, 2009.

CERIANI, R.; MEIRELLES, A. J. A. Predicting Vapor-Liquid Equilibria of Fatty Systems. Fluid Phase Equilib, n. 215, p. 227-236, 2004.

CERIANI, R.; PAIVA, F. R.; GONCALVES, C. B.; BATISTA, E. A. C.; MEIRELLES, A. J. Densities and Viscosities of Vegetable Oils of Nutritional Value. J. Chem. Eng. Data, n.53, p. 1846-1853, 2008.

CONSTANTINOU, L.; PRICKETT, S.E.; MAVROVOUNIOTIS, M.L. Estimation of Properties of Acyclic Organic Compounds Using Conjugation Operators. Ind. Eng. Chem. Res, n. 39, p. 395-402, 1994.

CUNHA, S.C.; OLIVEIRA, M.B.P.P. Discrimination of vegetable oils by triacylglycerols evaluation of profile using HPLC/ELSD. Food Chemistry, n. 95, p.518-524, 2006.

DDB (DORTMUND DATA BANK). Disponível em $<$ www.ddbst.com>. Acesso em fev. 2019.

DIPPR (DESIGN INSTITUTE FOR PHYSICAL PROPERTIES). DIPPR 801 Database. Disponível em $<$ www.dippr.byu.edu>. Acesso em fev. 2019.

DO CARMO, FR; EVANGELISTA, NS; FERNANDES, FAN; DE SANT'ANA, HB Avaliação de métodos ótimos para propriedades críticas e fator acêntrico de compostos de biodiesel com sua aplicação em Soave- Equações de Estado de Redlich-Kwong e Peng-Robinson J. Chem. Eng. Data, 60 (11), 3358, 2015.

DUNKLE, M. N.; DAVID, F.; SANDRA, P. Analysis of triglycerides in vegetable oils using the Agilent 1260 Infinity Analutical SFC System with evaporative light scattering detection. Agilent Technologies Publication, Number 5991-0987EN, 2012.

ELBRO, H. S.; FREDENSLUND, A.; RASMUSSEN, P. Group Contribution Method for the Prediction of Liquid Densities as a Function of Temperature for Solvents, Oligomers, and Polymers. Ind. Eng. Chem. Res, n. 30, p.2576-2582, 1991.

EVANGELISTA, N. S.; DO CARMO, F. R.; DE SANT'ANA, H. B. Estimation of Vapor Pressures and Enthalpies of Vaporization of Biodiesel-Related Fatty Acid Alkyl Esters. Part 1. Evaluation of Group Contribution and Corresponding States Methods. Ind. Eng. Chem. Res. 56, 2298- 2309, 2017a.

EVANGELISTA, N. S.; DO CARMO, F. R.; DE SANT'ANA, H. B. Estimation of Vapor Pressures and Enthalpies of Vaporization of Biodiesel-Related Fatty Acid Alkyl Esters. Part 2. New Parameters for Classic Vapor Pressure Correlations. Ind. Eng. Chem. Res., 56, 8349-8357, 2017b.

EVANGELISTA, N. S.; DO CARMO, F. R.; DE SANT'ANA, H. B. Estimation of Physical Constants of BiodieselRelated Fatty Acid Alkyl Esters: Normal Boiling Point, Critical Temperature, Critical Pressure, and Acentric Factor. Ind. Eng. Chem. Res. 57 (25), 8552-8565, 2018.

FALLEIRO, R. M. M; SILVA, L. Y. A.; MEIRELLES, A. J.A.; KRÄHENBÜHL, M. A. Vapor pressure data for fatty acids obtained using an adaptation of the DSC technique. Thermochimica Acta, n.547, p. 6-12, 2012.

FASINA, O. O.; COLLEY, Z. Viscosity and specific heat of 
vegetable oils as a function of temperature: $35^{\circ} \mathrm{C}$ to $180^{\circ} \mathrm{C}$. Int. J. Food Properties, 11 738-746, 2008.

FEDORS, R.F. A Relationship between Chemical Structure and the Critical Temperature. Chem. Eng. Comm., 1982.

FILHO, N. R. A.; MENDES, O. L.; LANCAS, F. M. Computer Prediction of Triacylglycerol Composition of Vegetable Oils by HRGC. J. Chromatogr., n. 40, p. 557-562, 1995.

FREDENSLUND, A.; JONES, R. L., PRAUSNITZ J. M. Group-Contribution Estimation of Activity Coefficients in Nonideal Liquid Mixtures. AIChE Journal, vol. 21, p. 1086, 1975.

GARCİA, M.; ALBA, J.-J.; GONZALO, A.; SANCHEZ, J. L.; ARAUZO, J. Comparison of Methods for Estimating Critical Properties of Alkyl Esters and Its Mixtures. J. Chem. Eng. Data, 58, 2689-2694, 2013.

GOODRUM, J. W; GELLER, D. P. Rapid thermogravimetric measurements of boiling points and vapor pressure of saturated medium- and long-chain triglycerides. Bioresource Technology, n. 84, p.75-80, 2002.

HALVORSEN, J. D.; MAMMEL, W. C., JR.; CLEMENTS, L. D. Density estimation for fatty acids and vegetable oils based on their fatty acid composition. J. Am. Oil Chem. Soc., n. 70, p. 875-880, 1993.

HAMMOND, E. G.; JOHNSON, L. A.; SU, C.; WANG, T.; WHITE, P. J. Soybean Oil. In: Bailey's Industrial Oil and Fat Products, Sixth Edition, Edited by Fereidoon Shahidi, John Wiley \& Sons, 2005.

IHMELS, E. C.; GMEHLING, J. Extension and Revision of the Group Contribution Method GCVOL for the Prediction of Pure Compound Liquid Densities. Ind. Eng. Chem. Res., n. 42, p.408-412. 2003.

JOBACK, K. G.; REID, R. C. Estimation of Pure-Component Properties from Group-Contributions. Chem. Eng. Comm, n. 57, p.233-243, 1987.

KESLER, M.G., LEE, B.I., Improve Predictions of Enthalpy of Fractions. Hydrocarbon Processing, n.55, p.153-158, 1976.

LI, J.; XIA, L.; XIANG, S. A New Method Based on Elements and Chemical Bonds for Organic Compounds Critical Properties Estimation. Fluid Phase Equilib., 417, 1-6, 2016.

LYDERSEN, A.L. Estimation of critical properties of organic compounds, College Engineering University Wisconsin, Engineering Experimental Station Report 3, Madison, WI, 1955.

MA, F., HANNA, M. A. Biodiesel production: a review. Bioresource Technology, 70(1), 1-15, 1999.

MARRERO, J.; GANI, R. Group-contribution based estimation of pure component properties. Fluid Phase Equilib., p.183-208, 2001.

MOLLER, B.; RAREY, J.; RAMJUGERNATH, D. Estimation of the Vapour Pressure of Non-Electrolyte Organic Compounds via Group Contributions and Group Interactions. J. Mol. Liq., 143 (1), 52, 2008.

MORAD, N. A.; KAMAL, A. A. M.; PANAU, F.; YEW, T. W. Liquid Specific Heat Capacity Estimation for Fatty Acids, Triacylglycerols, and Vegetable Oils Based on Their Fatty Acid Composition. J. Am. Oil Chem. Soc., 77, 1001-1005, 2000.

MURATA, K.; LIU, Y.; INABA, M.; TAKAHARA, I. Production of Synthetic Diesel by Hydrotreatment of Jatropha Oils Using Pt-Re/H-ZSM-5 Catalyst. Energy
Fuels, 24 (4):p.2404-9, 2010.

NANNOOLAL, Y.; RAREY, J.; RAMJUGERNATH, D. Estimation of Pure Component Properties Part 3. Estimation of the Vapor Pressure of Non-Electrolyte Organic Compounds via Group Contributions and Group Interactions. Fluid Phase Equilib., 269 (1-2), 117, 2008 .

NIKITIN, E. D.; PAVLOV, P. A.; SKRIPOV, P. V. Measurement of the critical properties of thermally unstable substances and mixtures by the pulse-heating method. J. Chem. Thermodynamics, 25, 869-880, 1993

NIKITIN, E. D.; POPOV, A. P. Vapor-Liquid Critical Point Measurements of Fifteen Compounds by the PulseHeating Method.Fluid Phase Equilib., 380, 11-17, 2014

NIST. National Institute of Standards and Technology. Disponível em: <http://www.nist.gov/>. Acesso em fev. 2019.

NOUREDDINI, H.; TEOH, B C.; CLEMENTS, L. D. Densities of Vegetable Oils and Fatty Acids. Papers in Biomaterials. Paper 14, 1992.

PERRY, E. S.; WEBER, W. H.; DAUBERT, B. F. Vapor Pressures of Phlegmatic Liquids. I. Simple and Mixed Triglycerides. J. Am. Chem. Soc., n.71 (11), p. 3720 3726, 1949.

PHILLIPS, J. C.; MATTAMAL, G. J. Effect of Number of Carboxyl Groups on Liquid Density of Esters of Alkylcarboxylic Acids. J. Chem. Eng., n. 23, p.1-6, 1978.

PITZER, K. S. The Volumetric and Thermodynamic Properties of Fluids. I. Theoretical Basis and Virial Coefficients. J. Am. Chem. Soc., n.77, p.3427-3433, 1955

POLING, B. E; PRAUSNITZ, J. M.; O'CONNELL, J. P. The Properties of Gases and Liquids. 5 ed. McGraw-Hill, New Yord, 803 p., 2001.

PRATAS, M. J.; FREITAS, S.; OLIVEIRA, M. B.; MONTEIRO, S. C.; LIMA, A. S.; COUTINHO, J. A. P Densities and viscosities of fatty acid methyl and ethyl esters. J. Chem. Eng., n.55, p.3983-3990, 2010.

REID, R. C.; PRAUSNITZ, J. M.; POLING, B. E. The properties of gases and liquids. 4th ed. New York: McGraw-Hill; 1987

RIO, J. C. D.; A. B. EVARISTO; MARQUES, G.; MARTÍNRAMOS, P.; MARTÍN-GIL, J.; GUTIÉRREZ, A. Chemical composition and thermal behavior of the pulp and kernel oils from macauba palm (Acrocomia aculeata) fruit. Industrial Crops and Products, 84, 294-304, 2016.

RODRIGUES, H. S. Obtenção de ésteres etílicos e metílicos, por reações de transesterificação, a partir do óleo da palmeira Latino Americana macaúba - Acrocomia aculeata. Tese de doutorado, Faculdade de Filosofia, Ciências e Letras de Riberão Preto da Universidade de São Paulo, Riberão Preto, São Paulo, 2007.

SALES-CRUZ, M.; ACA-ACA, G.; SÁNCHEZ-DAZA, O.; LÓPEZ-ARENAS, T. Predicting critical properties, density and viscosity of fatty acids, triacylglycerols and methyl esters by group contribution methods. 20th European Symposium on Computer Aided Process Engineering - ESCAPE20, 2010.

SANTANDER, C. M. G.; RUEDA, S. M. G.; SILVA, N. L., CAMARGO, C. L.; KIECKBUSCH, T. G.; MACIEL, 
M. R. W. Measurements of normal boiling points of fatty acid ethyl esters and triacylglycerols by thermogravimetric analysis. Fuel, n.92, p. 158-161, 2012.

SU, Y.-C.; LIU, Y. A.; DÍAZ-TOVAR, C.-A.; GANI, R. Selection of prediction methods for thermophysical properties for process modeling and product design of biodiesel manufacturing. Ind. Eng. Chem. Res., n. 50, p. 6809-6836, 2011 .

SUM, A. K.; BIDDY, M. J.; PABLO, J. J. Predictive Molecular Model for the Thermodynamic and Transport Properties of Triacylglycerols. J. Phys. Chem., n. 107, p.1444314451, 2003.

VATANI, A., MEHRPOOYA, M. E GHARAGHEIZI, F. Prediction of Standard Enthalpy of Formation by a QSPR
Model. Int. J. Mol. Sci., n. 8, p.407-432, 2007.

WALLEK, T.; RAREY, J.; METZGER, J. O.; GMEHLING, J. Estimation of Pure-Component Properties of BiodieselRelated Components: Fatty Acid Methyl Esters, Fatty Acids, and Triglycerides. Ind. Eng. Chem. Res., n.52, p.16966-16978, 2013.

ZONG, L.; RAMANATHAN, S.; CHEN, C. C. Predicting Thermophysical Properties of Mono- and Diglycerides with the Chemical Constituent Fragment Approach. Ind. Eng. Chem. Res., n.49, p. 5479-5484, 2010 b.

ZONG, L.; RAMANATHAN, S.; CHEN, C.-C. Fragment-based approach for estimating thermophysical properties of fats and vegetable oils for modeling biodiesel production processes. Ind. Eng. Chem. Res., n.49, p. 876-886, 2010a. 